\title{
Triplet Energy Back Transfer in Conjugated Polymers with Pendant Phosphorescent Iridium Complexes
}

\author{
Nicholas R. Evans,,$^{\dagger}$ Lekshmi Sudha Devi, ${ }^{+}$Chris S. K. Mak, ${ }^{\dagger}$ Scott E. Watkins, ${ }^{\dagger,}$ Sofia I. Pascu, ${ }^{\dagger}$ \\ Anna Köhler, ${ }^{t, \|}$ Richard H. Friend, ${ }^{+}$Charlotte K. Williams ${ }^{\dagger, \perp}$ and Andrew B. Holmes $* \xi, \perp$
}

Contribution from Melville Laboratory for Polymer Synthesis, Department of Chemistry, University of Cambridge, Lensfield Road, Cambridge CB2 1EW, United Kingdom, Cavendish Laboratory, Department of Physics, University of Cambridge, Madingley Road, Cambridge CB3 0HE, United Kingdom, School of Chemistry, University of Melbourne, Victoria 3010, Australia, Institute of Physics, University of Potsdam, Am Neuen Palais, Potsdam 14469, Germany and Department of Chemistry, Imperial College, South Kensington, London, SW7 2AZ, United Kingdom.

E-mail: aholmes@unimelb.edu.au 


\section{Truncated References from Article}

(25) Friend, R. H.; Gymer, R. W.; Holmes, A. B.; Burroughes, J. H.; Marks, R. N.; Taliani, C.;

Bradley, D. D. C.; Dos Santos, D. A.; Brédas, J. L.; Lögdlund, M.; Salaneck, W. R. Nature 1999, 397, $121-128$.

(34) Zhang, M.; Lu, P.; Wang, X.; He, L.; Xia, H.; Zhang, W.; Yang, B.; Liu, L.; Yang, L.; Yang, M.; Ma, Y.; Feng, J.; Wang, D.; Tamai, N. J. Phys. Chem. B 2004, 108, 13185-13190.

(51) Niu, Y.-H.; Chen, B.; Liu, S.; Yip, H.; Bardecker, J.; Jen, A. K.-Y.; Kavitha, J.; Chi, Y.; Shu, C.F.; Tseng, Y.-H.; Chien, C.-H. Appl. Phys. Lett. 2004, 85, 1619-1621. 


\section{General Experimental Methods}

Optical measurements: Solutions of the materials were prepared at a concentration of typically 15 $\mathrm{mg} \mathrm{ml}^{-1}$ in toluene. Thin films of the materials were obtained by spin-coating onto quartz. The absorption spectra were taken using a Hewlett-Packard ultraviolet-visible (UV-VIS) absorption spectrometer. The room temperature photoluminescence spectra were recorded with the samples under vacuum using a spectrograph with an optical fiber input coupled to a cooled charge coupled device (CCD) parallel detection system (Oriel IntraspecIV). The excitation was provided by the UV lines of a continuous wave (cw) argon ion laser (355 nm and $364 \mathrm{~nm})$ with a typical intensity of $25 \mathrm{~mW} \mathrm{~mm}^{-2}$. Measurements of the overall photoluminescence quantum yield were taken at room temperature using an integrating sphere with the same CCD/Oriel Intraspect IV spectrograph. Excitation was taken from the $\mathrm{cw}$ argon ion laser at $364 \mathrm{~nm}$ with an excitation density of about $1 \mathrm{~mW} \mathrm{~mm}$. The lifetime of the phosphorescence emission from the samples under vacuum were measured by exciting the samples with $355 \mathrm{~nm}$ light pulses from a Q-switched Nd:YAG laser. The emitted light was detected using a photomultiplier tube and its temporal response was viewed on an oscilloscope.

Device fabrication and electroluminescence measurements: A single-emissive layer configuration was employed for device fabrication: glass/ITO/PEDOT:PSS/copolymer/Ca/Al. A PEDOT:PSS layer with a typical thickness of $20 \mathrm{~nm}$ was spin-coated from aqueous solution onto pre-patterned ITO substrates previously treated with oxygen plasma. The active light-emitting layer with a thickness of $100 \mathrm{~nm}$ was spin-coated from toluene solution under a nitrogen atmosphere. A $20 \mathrm{~nm}$ thick Ca layer served as the cathode with a $100 \mathrm{~nm}$ thick $\mathrm{Al}$ cap. The electroluminescence measurements were carried out under vacuum. The electroluminescence spectra were taken by the same detection system as described for photoluminescence. The driving voltage is applied by a Keithley 2400 sourcemeter. Current-voltage-luminance characteristics were recorded using a Keithley 230 programmable voltage source, a Keithley 617 programmable electrometer and a photodiode. 
Reagents: Pentane-2,4-dione 5 and diisopropylamine were purified by published procedures. ${ }^{1}$ Crude di- $\mu$-chloro- $N, N^{\prime}$-trans- $N^{\prime \prime}, N^{\prime \prime \prime}$-trans-tetrakis[2-(2-pyridyl- $\left.\kappa N\right)$ benzo[b]thien-3-yl]diiridium(III) $\quad 7,{ }^{2}$ 9,9dioctyl-2,7-bis(4,4,5,5-tetramethyl-1,3,2-dioxaborolan-2-yl)-9H-fluorene $14,{ }^{3}$ 2,7-dibromo-9,9-dioctyl9H-fluorene $15{ }^{3}$ and 4-methoxy-6-methyl-2H-pyran-2-one ${ }^{4}$ were prepared by following the published procedures. All other reagents were used as received from commercial suppliers.

Solvents: THF was distilled from calcium hydride and lithium aluminium hydride. Toluene was distilled from calcium hydride. Tetrachloromethane was distilled from calcium chloride. Other solvents were distilled or used as received from commercial suppliers.

Reactions were degassed and performed under an inert atmosphere unless specified otherwise. Water-sensitive reactions were conducted in oven- or flame-dried glassware. The synthesis of 9-octyl9H-fluorene 2 was performed in a $100 \mathrm{~cm}^{3}$ Chemware PFA (perfluoroalkoxy) Erlenmeyer flask (SaintGobain Performance Plastics). Temperatures refer to the bath temperature. Brine is saturated aqueous sodium chloride.

Dry flash chromatography ${ }^{5}$ was performed using Merck 9385 silica gel 60 (0.040-0.063 mm).

Kugelrohr distillations were conducted in a Büchi GKR-51 glass oven and temperatures refer to the oven temperature.

Yields refer to isolated quantities of analytically pure material unless specified otherwise.

Melting points below $200{ }^{\circ} \mathrm{C}$ were determined with a Reichert hot-stage microscope and are uncorrected. Melting points above $200{ }^{\circ} \mathrm{C}$ were determined with an Electrothermal Engineering IA9100 apparatus and are uncorrected. The crystallization solvent is given in parentheses.

Elemental analyses were performed by the University Chemical Laboratory's microanalysis service with an Exeter Analytical CE-440 elemental analyzer.

Retention factors were measured on cut Merck 5715 silica gel $60 \mathrm{~F}_{254}$ TLC plates (layer thickness $0.25 \mathrm{~mm}$ ), observed under $254 \mathrm{~nm}$ or $366 \mathrm{~nm}$ ultraviolet light or after thermal development with an alkaline potassium permanganate solution. The eluent is given in parentheses. 
Ultraviolet and visible absorptions were measured for dilute (absorbance below unity) chloroform solutions in a $1.0 \mathrm{~cm}$ pathlength quartz cuvette on a Hewlett-Packard 8452A diode array spectrophotometer. Molar extinction coefficients $\left(1000 \mathrm{~cm}^{2} \mathrm{~mol}^{-1}\right)$ are given in the logarithm to base ten. Shoulders are denoted by 'sh'.

Infrared absorptions were measured for chloroform solutions in a $1.0 \mathrm{~mm}$ pathlength sodium chloride sealed cell with a PerkinElmer Spectrum RX I FT-IR spectrometer or a Nicolet 510 FT-IR spectrometer.

Proton NMR spectra were recorded on Bruker DPX-250 (250 MHz), Bruker DPX-400 (400 MHz), Bruker DRX-400 (400 MHz) or Bruker DRX-500 (500 MHz) instruments. Double-quantum filtered gradient COSY spectra were recorded on a Bruker DRX-400 (400 MHz) instrument. Chemical shifts are reported in ppm relative to residual protons in the commercially available deuterated solvents $\mathrm{CDCl}_{3}$ ( $\delta 7.26)$ or $d_{6}$-acetone $(\delta 2.04)$. The number of equivalent nuclei (by integration), multiplicity [s (singlet), br s (broad singlet), d (doublet), $\mathrm{t}$ (triplet), dd (doublet of doublets), dt (doublet of triplets), $\mathrm{m}$ (multiplet) or br m (broad multiplet)], coupling constants where appropriate (mean values for mutually coupled protons) and assignments [Duplicated ring systems are appended with a letter ('A', 'B', 'C' or 'D') where necessary. Subscripts on locants denote a methylene ('2'), methyl ('3’) or one proton of a diastereotopic methylene ('gem')] are given in parentheses.

Carbon-13 NMR spectra were recorded with proton decoupling on Bruker DRX-400 (100 MHz) or Bruker DRX-500 Dual CryoProbe (125 MHz) instruments. Chemical shifts are reported in ppm relative to $\mathrm{CDCl}_{3}(\delta 77.0)$. The latent multiplicities due to proton coupling [s (singlet), $\mathrm{d}$ (doublet), $\mathrm{t}$ (triplet) or $\mathrm{q}$ (quartet)] as determined by an Attached Proton Test (APT) (and, where necessary, a Distortionless Enhancement by Polarization Transfer (DEPT) $135^{\circ}$ ) experiment are given in parentheses.

Mass spectrometry was performed by the EPSRC National Mass Spectrometry Service, Swansea. Low resolution spectra were recorded on a Waters Micromass Quattro II instrument (EI) or an Applied Biosystems Voyager-DE STR (MALDI). Accurate mass measurements were made on a Thermo 
Electron Finnigan MAT900XLT (EI and ES) for compounds of molecular weight below 1100. Relative intensities are given in parentheses. (For the copolymers 17 and 18, the number-average, $\bar{M}_{\mathrm{n}}$, and weight-average, $\bar{M}_{\mathrm{w}}$, molecular weights and the polydispersity index, PDI, are given. The ions observed are listed with the number fractions and assignments (where $F$ is a fluorenyl repeat unit, $I r$ is a fluorenyl repeat unit bonded with an iridium complex, and $P h$ and $H$ are phenyl and proton end-groups, respectively) in parentheses.)

Gel permeation chromatography was performed by eluting $0.1 \mathrm{~cm}^{3}$ injection volume of a polymer solution ( $1 \mathrm{mg} \mathrm{cm}^{-3}$ in THF) through two Polymer Laboratories PLgel $5 \mu \mathrm{m}$ MIXED-C columns in series at $30{ }^{\circ} \mathrm{C}$ with THF at a flowrate of $1 \mathrm{~cm}^{3} \mathrm{~min}^{-1}$. The eluate was monitored with a Viscotek Model 200 differential refractometer and the number-average, $\bar{M}_{\mathrm{n}}$, peak, $M_{\mathrm{p}}$, and weight-average, $\bar{M}_{\mathrm{w}}$, molecular weights and the polydispersity index, PDI, are referenced against a calibration with Polymer Laboratories PS-1 polystyrene standards.

Differential scanning calorimetry was performed using a PerkinElmer Pyris 1 differential scanning calorimeter with liquid nitrogen as coolant. Samples of $c a .5 \mathrm{mg}$ of polymer were compressed in aluminium pans and crimped. The samples were heated, cooled and heated again between $0{ }^{\circ} \mathrm{C}$ and 300 ${ }^{\circ} \mathrm{C}$ at a rate of $200{ }^{\circ} \mathrm{C} \mathrm{min}{ }^{-1}$ under a $20 \mathrm{~cm}^{3} \mathrm{~min}^{-1}$ flowrate of helium. The first heating step afforded determination of the glass-transition temperature range, $T_{\mathrm{g}}$, and the second heating step the peak maximum crystallization, $T_{\mathrm{c}}$, and melting, $T_{\mathrm{m}}$, temperatures.

Thermogravimetric analyses were performed with a PerkinElmer Pyris 1 thermogravimetric analyzer by heating from $50{ }^{\circ} \mathrm{C}$ to $1000{ }^{\circ} \mathrm{C}$ at a rate of $20{ }^{\circ} \mathrm{C} \mathrm{min}-1$ under a $20 \mathrm{~cm}^{3} \mathrm{~min}^{-1}$ flowrate of nitrogen. The trigger-point decomposition temperature for $0.5 \%$ mass loss, $T_{\mathrm{d}}$, is reported and a brief description of the decomposition trace is given in parentheses. 


\section{Synthetic Procedures and Characterization}

\section{9-Octyl-9H-fluorene 2}

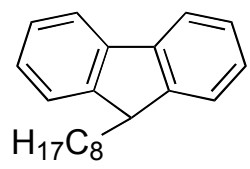

A mixture of $9 H$-fluorene $1(10.0 \mathrm{~g}, 60.2 \mathrm{mmol})$, finely ground potassium hydroxide $(1.69 \mathrm{~g}, 30.1$ mmol) and octan-1-ol (40g, $0.31 \mathrm{~mol})$ was stirred for $19 \mathrm{~h}$ in a $100 \mathrm{~cm}^{3}$ Chemware PFA (perfluoroalkoxy) Erlenmeyer flask at $190{ }^{\circ} \mathrm{C}$ under air. The mixture was cooled to RT before octan-1ol was distilled from the mixture $\left(56-59^{\circ} \mathrm{C}, 0.7 \mathrm{mmHg}\right)$. The crude product was purified by dry flash chromatography using hexane as eluent to give 9-octyl-9H-fluorene $\mathbf{2}(16.0 \mathrm{~g}, 96 \%)$ as a colorless liquid that crystallized upon cooling to $0{ }^{\circ} \mathrm{C}$ as a white solid, mp $33-35{ }^{\circ} \mathrm{C}$.

Anal. Calcd for $\mathrm{C}_{21} \mathrm{H}_{26}$ : C, 90.6; H, 9.4. Found: C, 90.7; H, 9.3. $R_{\mathrm{f}}$ (hexane) 0.3. IR $\left(\mathrm{CHCl}_{3}\right) v_{\max }$ : 2929, 2857, $1450 \mathrm{~cm}^{-1} .{ }^{1} \mathrm{H}$ NMR (400 MHz, $\left.\mathrm{CDCl}_{3}, \delta\right): 0.95\left(\mathrm{t}, J=7.0 \mathrm{~Hz}, 3 \mathrm{H}, \mathrm{CH}_{3}\right), 1.23-1.40(\mathrm{~m}, 12$ $\left.\mathrm{H}, 6 \times \mathrm{CH}_{2}\right), 2.03-2.11\left(\mathrm{~m}, 2 \mathrm{H}, \mathrm{CH}_{2}\right), 4.04(\mathrm{t}, J=5.9 \mathrm{~Hz}, 1 \mathrm{H}, 9-\mathrm{H}), 7.37(\mathrm{t}, J=7.2 \mathrm{~Hz}, 2 \mathrm{H}, 2 \times \mathrm{ArH})$, $7.43(\mathrm{t}, J=7.2 \mathrm{~Hz}, 2 \mathrm{H}, 2 \times \mathrm{ArH}), 7.58(\mathrm{~d}, J=7.2 \mathrm{~Hz}, 2 \mathrm{H}, 2 \times \mathrm{ArH}), 7.82(\mathrm{~d}, J=7.2 \mathrm{~Hz}, 2 \mathrm{H}, 2 \times$ ArH). ${ }^{13} \mathrm{C} \mathrm{NMR}\left(\mathrm{CDCl}_{3}, \delta\right): 14.1(\mathrm{q}), 22.6(\mathrm{t}), 25.7(\mathrm{t}), 29.3(\mathrm{t}), 29.4(\mathrm{t}), 30.0(\mathrm{t}), 31.8(\mathrm{t}), 33.1(\mathrm{t}), 47.5$ (d), 119.7 (d), 124.3 (d), 126.7 (d), 126.8 (d), 141.1 (s), 147.6 (s). HRMS-EI (m/z): $\mathrm{M}^{+}$calcd for $\mathrm{C}_{21} \mathrm{H}_{26}$, 278.2035; found, 278.2033. MS-EI m/z (\% relative intensity): 278 (47), 178 (33), 165 (100).

\section{2,7-Dibromo-9-octyl-9H-fluorene $3^{6}$}

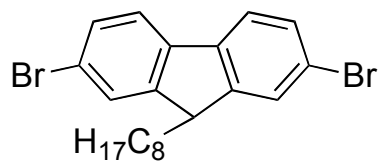

Bromine $\left(0.8 \mathrm{~cm}^{3}, 0.02 \mathrm{~mol}\right)$ was added dropwise over $7 \mathrm{~min}$ to a stirred solution of 9-octyl-9Hfluorene $2(2.11 \mathrm{~g}, 7.57 \mathrm{mmol})$ and iodine $(0.15 \mathrm{~g}, 0.61 \mathrm{mmol})$ in DCM $\left(15 \mathrm{~cm}^{3}\right)$ at $0{ }^{\circ} \mathrm{C}$ in the dark. The mixture was stirred for $2 \mathrm{~h}$ at $0{ }^{\circ} \mathrm{C}$ in the dark and then for $2.5 \mathrm{~h}$ at $\mathrm{RT}$ in the dark after which it was 
quenched with aqueous sodium thiosulfate $\left(20 \% ; 5 \mathrm{~cm}^{3}\right)$. The aqueous phase was separated and extracted with DCM $\left(4 \times 50 \mathrm{~cm}^{3}\right)$ and the combined organic phases were dried over magnesium sulfate and evaporated under reduced pressure. The crude product was purified by dry flash chromatography using hexane-DCM (1:0 changing to 0:1) as eluent to give 2,7-dibromo-9-octyl-9H-fluorene 3 (2.98 g, $90 \%$ ) as a white solid, mp $75-79^{\circ} \mathrm{C}$.

Anal. Calcd for $\mathrm{C}_{21} \mathrm{H}_{24} \mathrm{Br}_{2}$ : C, 57.8; H, 5.6. Found: C, 57.9; H, 5.5. $R_{\mathrm{f}}$ (hexane) 0.4. IR $\left(\mathrm{CHCl}_{3}\right) v_{\max }$ : 2929, 2858, 1454, $1062 \mathrm{~cm}^{-1} .{ }^{1} \mathrm{H}$ NMR $\left(250 \mathrm{MHz}, \mathrm{CDCl}_{3}, \delta\right): 0.87\left(\mathrm{t}, J=6.7 \mathrm{~Hz}, 3 \mathrm{H}, \mathrm{CH}_{3}\right), 1.06-1.35$ (m, $\left.12 \mathrm{H}, 6 \times \mathrm{CH}_{2}\right), 1.90-2.04\left(\mathrm{~m}, 2 \mathrm{H}, \mathrm{CH}_{2}\right), 3.95(\mathrm{t}, J=5.8 \mathrm{~Hz}, 1 \mathrm{H}, 9-\mathrm{H}), 7.49(\mathrm{dd}, J=1.7 \mathrm{~Hz}$ and 8.1 Hz, $2 \mathrm{H}, 3-\mathrm{H}$ and 6-H), $7.56(\mathrm{~d}, J=8.1 \mathrm{~Hz}, 2 \mathrm{H}, 4-\mathrm{H}$ and 5-H), $7.62(\mathrm{~d}, J=1.7 \mathrm{~Hz}, 2 \mathrm{H}, 1-\mathrm{H}$ and 8H). ${ }^{13} \mathrm{C} \mathrm{NMR}\left(\mathrm{CDCl}_{3}, \delta\right): 14.0(\mathrm{q}), 22.6(\mathrm{t}), 25.4(\mathrm{t}), 29.2(\mathrm{t}), 29.2(\mathrm{t}), 29.7(\mathrm{t}), 31.7(\mathrm{t}), 32.6(\mathrm{t}), 47.4(\mathrm{~d})$, 121.0 (d), 121.1 (s), 127.6 (d), 130.1 (d), 139.0 (s), 149.2 (s). HRMS-EI (m/z): $\mathrm{M}^{+}$calcd for $\mathrm{C}_{21} \mathrm{H}_{24}{ }^{79} \mathrm{Br}_{2}$, 434.0245; found, 434.0242. MS-EI m/z (\% relative intensity): 436 (40), 323 (26), 276 (20), 243 (56), 178 (37), $163(66), 57(100)$.

\section{2,7-Dibromo-9-(8-bromooctyl)-9-octyl-9H-fluorene 4}

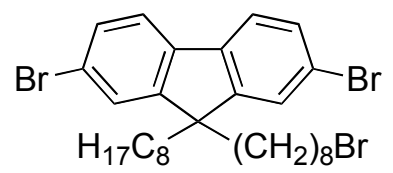

A mixture of 2,7-dibromo-9-octyl-9H-fluorene 3 (2.63 g, $6.03 \mathrm{mmol}), 1,8$-dibromooctane (16.4 g, $60.3 \mathrm{mmol})$, aqueous sodium hydroxide $\left(33 \% ; 20 \mathrm{~cm}^{3}\right)$ and Aliquat 336 (2 drops) was stirred vigorously for $1 \mathrm{~d}$ at $80^{\circ} \mathrm{C}$. The mixture was cooled to RT and extracted with DCM $\left(3 \times 50 \mathrm{~cm}^{3}\right)$. The combined extracts were dried over magnesium sulfate and evaporated under reduced pressure. The excess 1,8 -dibromooctane was recovered from the mixture by Kugelrohr distillation $\left(180{ }^{\circ} \mathrm{C}, 0.1 \mathrm{mmHg}\right)$. The crude product was purified by dry flash chromatography using hexane-DCM (1:0 changing to 4:1) as eluent to give the tribromide $4(2.67 \mathrm{~g}, 71 \%)$ as a colorless oil. 
Anal. Calcd for $\mathrm{C}_{29} \mathrm{H}_{39} \mathrm{Br}_{3}$ : C, 55.5; H, 6.3. Found: C, 55.8; H, 6.4. $R_{\mathrm{f}}$ (hexane) 0.2. IR $\left(\mathrm{CHCl}_{3}\right) v_{\max }$ : 2931, 2858, 1600, $1461 \mathrm{~cm}^{-1} .{ }^{1} \mathrm{H}$ NMR $\left(400 \mathrm{MHz} \mathrm{CDCl}_{3}, \delta\right): 0.52-0.64\left(\mathrm{~m}, 4 \mathrm{H}, 2 \times \mathrm{CH}_{2}\right), 0.83(\mathrm{t}, J=$ 7.1 Hz, $\left.3 \mathrm{H}, \mathrm{CH}_{3}\right), 1.00-1.25\left(\mathrm{~m}, 16 \mathrm{H}, 8 \times \mathrm{CH}_{2}\right), 1.26-1.35\left(\mathrm{~m}, 2 \mathrm{H}\right.$, bromooctyl 6- $\left.\mathrm{H}_{2}\right), 1.73-1.81(\mathrm{~m}, 2$ $\mathrm{H}$, bromooctyl 7- $\left.\mathrm{H}_{2}\right), 1.87-1.96\left(\mathrm{~m}, 4 \mathrm{H}, 2 \times \mathrm{CH}_{2}\right), 3.35\left(\mathrm{t}, \mathrm{J}=6.9 \mathrm{~Hz}, 2 \mathrm{H}\right.$, bromooctyl 8- $\left.\mathrm{H}_{2}\right), 7.42-$ $7.47(\mathrm{~m}, 4 \mathrm{H}, 4 \times \mathrm{ArH}), 7.52(\mathrm{~d}, J=7.9 \mathrm{~Hz}, 2 \mathrm{H}, 2 \times \mathrm{ArH}) .{ }^{13} \mathrm{C} \mathrm{NMR}\left(\mathrm{CDCl}_{3}, \delta\right): 14.0(\mathrm{q}), 22.5(\mathrm{t}), 23.4$ $(\mathrm{t}), 23.5(\mathrm{t}), 27.9(\mathrm{t}), 28.4(\mathrm{t}), 28.8(\mathrm{t}), 29.0(\mathrm{t}), 29.1(\mathrm{t}), 29.6(\mathrm{t}), 29.7(\mathrm{t}), 31.7(\mathrm{t}), 32.6(\mathrm{t}), 33.8(\mathrm{t}), 40.0$ (t), $40.1(\mathrm{t}), 55.6(\mathrm{~s}), 121.0(\mathrm{~d}), 121.4(\mathrm{~s}), 126.1(\mathrm{~d}), 130.1(\mathrm{~d}), 139.0(\mathrm{~s}), 152.4(\mathrm{~s})$. HRMS-EI $(\mathrm{m} / \mathrm{z}): \mathrm{M}^{+}$ calcd for $\mathrm{C}_{29} \mathrm{H}_{39}{ }^{79} \mathrm{Br}_{3}, 624.0602$; found, 624.0609. MS-EI $m / z$ (\% relative intensity): 628 (35), 323 (40), $176(68), 82(56), 69(37), 57(68)$.

\section{3-(2,7-Dibromo-9-octyl-9H-fluoren-9-yl)tridecane-2,4-dione 6}

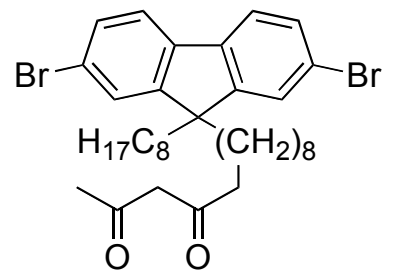

Sodium hydride $(60 \%$ dispersion in mineral oil; $320 \mathrm{mg}, 8.00 \mathrm{mmol})$ was washed with THF $(2 \times 15$ $\left.\mathrm{cm}^{3}\right)$. Pentane-2,4-dione $5\left(0.80 \mathrm{~cm}^{3}, 7.8 \mathrm{mmol}\right)$ was added dropwise over $20 \mathrm{~min}$ to a stirred suspension of the sodium hydride in THF $\left(20 \mathrm{~cm}^{3}\right)$ at $0{ }^{\circ} \mathrm{C}$. The resultant mixture was stirred for $30 \min$ at $0{ }^{\circ} \mathrm{C}$. Butyllithium (1.6 $\mathrm{M}$ in hexane; $\left.4.9 \mathrm{~cm}^{3}, 7.8 \mathrm{mmol}\right)$ was added dropwise over 20 min to the stirred mixture at $0{ }^{\circ} \mathrm{C}$. The resultant solution was stirred for $25 \mathrm{~min}$ at $0{ }^{\circ} \mathrm{C}$. A solution of 2,7-dibromo-9-(8bromooctyl)-9-octyl-9H-fluorene 4 (1.62 g, $2.58 \mathrm{mmol})$ in THF $\left(5 \mathrm{~cm}^{3}\right)$ was added dropwise over 10 min to the stirred solution at $0{ }^{\circ} \mathrm{C}$. The resultant mixture was stirred for $1 \mathrm{~h}$ at $0{ }^{\circ} \mathrm{C}$ and for a further $1 \mathrm{~h}$ at RT, after which it was quenched with aqueous ammonium chloride $\left(10 \% ; 8 \mathrm{~cm}^{3}\right)$. The mixture was acidified with concentrated hydrochloric acid to $\mathrm{pH} 1$ and the aqueous phase was separated and extracted with diethyl ether $\left(2 \times 15 \mathrm{~cm}^{3}\right)$. The combined organic phases were washed with saturated 
aqueous sodium bicarbonate $\left(2 \times 5 \mathrm{~cm}^{3}\right)$, dried with brine $\left(5 \mathrm{~cm}^{3}\right)$ and magnesium sulfate and evaporated under reduced pressure. The crude product was purified by dry flash chromatography using hexane-DCM (1:0 changing to $0: 1)$ as eluent and Kugelrohr distillation $\left(250{ }^{\circ} \mathrm{C}, 0.05 \mathrm{mmHg}\right)$ to give the tethered ligand $\mathbf{6}(969 \mathrm{mg}, 58 \%)$ as a pale yellow solid, $\mathrm{mp} 55-57^{\circ} \mathrm{C}$.

Anal. Calcd for $\mathrm{C}_{34} \mathrm{H}_{46} \mathrm{Br}_{2} \mathrm{O}_{2}$ : C, 63.2; H, 7.2. Found: C, 63.3; H, 7.0. $R_{\mathrm{f}}(\mathrm{DCM})$ 0.5. IR $\left(\mathrm{CHCl}_{3}\right) v_{\max }$ : 2931, 2856, 1619br, $1461 \mathrm{~cm}^{-1} .{ }^{1} \mathrm{H}$ NMR (400 MHz, $\left.\mathrm{CDCl}_{3}, \delta\right): 0.52-0.64\left(\mathrm{~m}, 4 \mathrm{H}, 2 \times \mathrm{CH}_{2}\right), 0.83(\mathrm{t}, J$ $\left.=7.1 \mathrm{~Hz}, 3 \mathrm{H}, \mathrm{CH}_{3}\right), 0.96-1.29\left(\mathrm{~m}, 20 \mathrm{H}, 10 \times \mathrm{CH}_{2}\right), 1.47-1.59\left(\mathrm{~m}, 2 \mathrm{H}\right.$, tridecyl 6- $\left.\mathrm{H}_{2}\right), 1.87-1.94(\mathrm{~m}, 4$ $\left.\mathrm{H}, 2 \times \mathrm{CH}_{2}\right), 2.04\left(\mathrm{~s}, 2.4 \mathrm{H}\right.$, enolone 1-H $\left.\mathrm{H}_{3}\right), 2.22\left(\mathrm{~s}, 0.6 \mathrm{H}\right.$, dione 1- $\left.\mathrm{H}_{3}\right), 2.22(\mathrm{t}, J=7.6 \mathrm{~Hz}, 1.6 \mathrm{H}$,

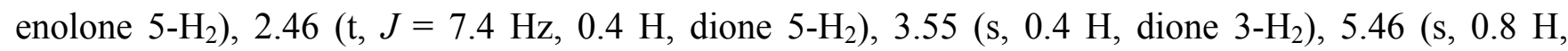
enolone 3-H), 7.42-7.47 (m, $4 \mathrm{H}, 4 \times \mathrm{ArH}), 7.51(\mathrm{~d}, J=8.0 \mathrm{~Hz}, 2 \mathrm{H}, 2 \times \mathrm{ArH}), 15.48$ (br s, $0.8 \mathrm{H}$, enolone $\mathrm{OH}) .{ }^{13} \mathrm{C} \mathrm{NMR}\left(\mathrm{CDCl}_{3}, \delta\right): 14.1(\mathrm{q}), 22.6(\mathrm{t}), 23.3(\mathrm{t}), 23.6(\mathrm{t}), 23.6(\mathrm{t}), 25.0(\mathrm{q}), 25.6(\mathrm{t}), 28.9$ $(\mathrm{t}), 29.1(\mathrm{t}), 29.1(\mathrm{t}), 29.1(\mathrm{t}), 29.2(\mathrm{t}), 29.2(\mathrm{t}), 29.3(\mathrm{t}), 29.8(\mathrm{t}), 29.8(\mathrm{t}), 30.8(\mathrm{q}), 31.7(\mathrm{t}), 38.2(\mathrm{t}), 40.1$ $(\mathrm{t}), 40.1(\mathrm{t}), 43.8(\mathrm{t}), 55.7(\mathrm{~s}), 57.9(\mathrm{t}), 99.7(\mathrm{~d}), 121.1(\mathrm{~d}), 121.5(\mathrm{~s}), 126.1(\mathrm{~d}), 130.1(\mathrm{~d}), 139.0(\mathrm{~s})$, 152.5 (s), 191.4 (s), 194.2 (s), 202.1 (s), 204.2 (s). HRMS-EI (m/z): $\mathrm{M}^{+}$calcd for $\mathrm{C}_{34} \mathrm{H}_{46}{ }^{79} \mathrm{Br}_{2} \mathrm{O}_{2}$, 644.1859; found, 644.1859. MS-EI m/z (\% relative intensity): 646 (16), 566 (7), 323 (22), 243 (25), 176 (31), 135 (7), $100(15), 85(100), 69(26), 57(86)$. 


\section{[13-(2,7-Dibromo-9-octyl-9H-fluoren-9-yl)tridecane-2,4-dionato]- $N, N^{\prime}$-trans-bis[2-(2-pyridyl-}

\section{$\kappa N)$ benzo[b]thien-3-yl]iridium(III) 8}

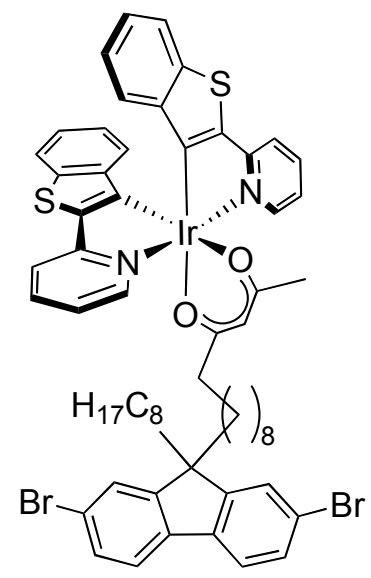

A mixture of 13-(2,7-dibromo-9-octyl-9H-fluoren-9-yl)tridecane-2,4-dione 6 (55 mg, $85 \mu \mathrm{mol})$, crude di- $\mu$-chloro- $N, N^{\prime}$-trans- $N^{\prime \prime}, N^{\prime \prime \prime}$-trans-tetrakis[2-(2-pyridyl- $\left.\kappa N\right)$ benzo[b]thien-3-yl]diiridium(III) $7 \quad$ (52 $\mathrm{mg}, c a .40 \mu \mathrm{mol})$ and sodium carbonate $(43 \mathrm{mg}, 0.40 \mathrm{mmol})$ in 2-ethoxyethanol $\left(4 \mathrm{~cm}^{3}\right)$ was stirred for $19 \mathrm{~h}$ under reflux. The mixture was cooled to RT, diluted with water $\left(15 \mathrm{~cm}^{3}\right)$ and the solvents were evaporated under reduced pressure. 2-(Benzo[b]thien-2-yl)pyridine was sublimed from the mixture at $\left(120{ }^{\circ} \mathrm{C}, 0.1 \mathrm{mmHg}\right)$ before the crude product was purified by dry flash chromatography using hexaneDCM (1:0 changing to 3:2) as eluent. The product was taken up in hexane-DCM and the solvents were evaporated under reduced pressure to leave an inseparable mixture of the tethered monomer $\mathbf{8}$ and its hydrodebrominated derivatives $(53 \mathrm{mg})$ as a red powder.

Anal. Calcd for $\mathrm{C}_{60} \mathrm{H}_{61} \mathrm{Br}_{2} \mathrm{IrN}_{2} \mathrm{O}_{2} \mathrm{~S}_{2}$ : C, 57.3; H, 4.9; N, 2.2. Found: C, 60.5; H, 5.4; N, 2.2. $R_{\mathrm{f}}(\mathrm{DCM})$ 0.8. A similar (half-scale) experiment was performed at $80^{\circ} \mathrm{C}$ and the product was also found to exhibit a signal characteristic of the hydrodebrominated derivatives $(\delta 7.30-7.35)$ upon analysis by proton NMR, as illustrated in Figure S1. 


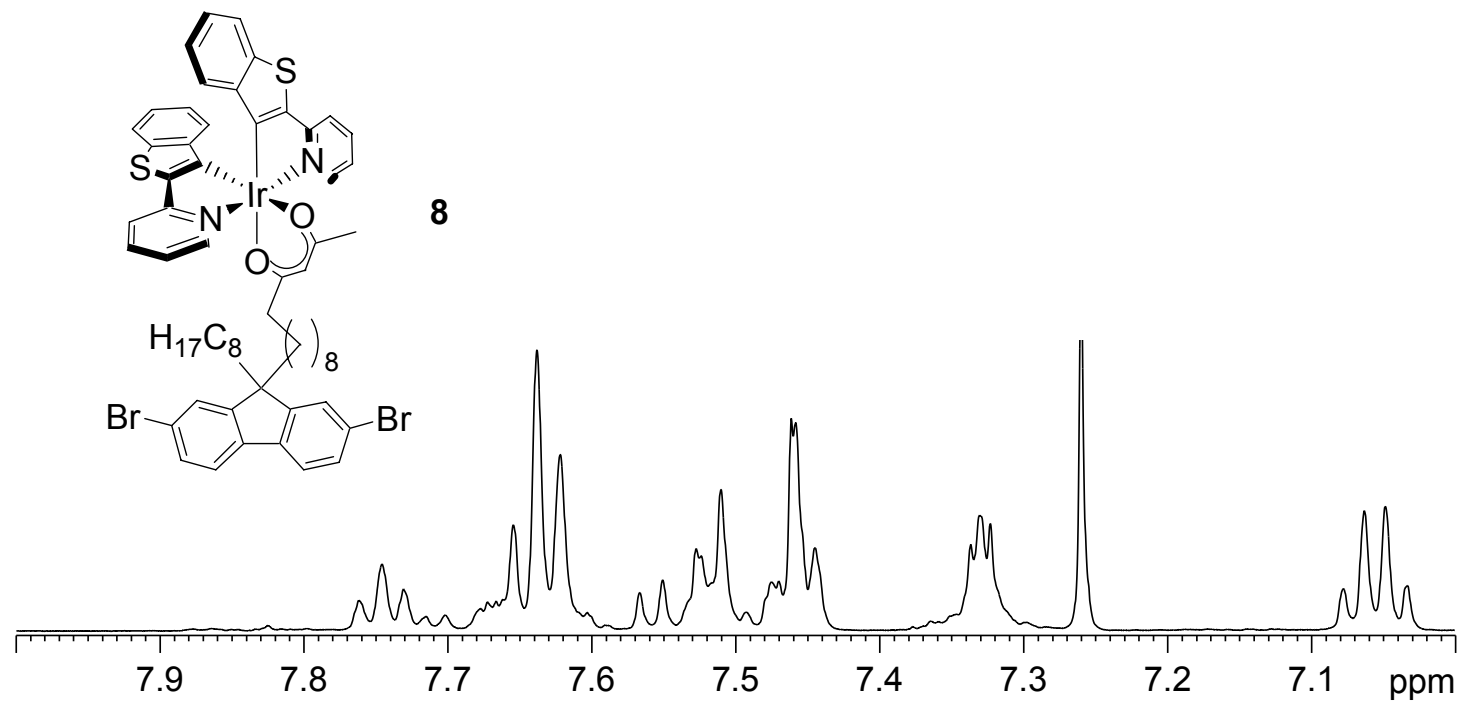

Figure S1. Detail from the aromatic region of a proton NMR spectrum (500 MHz, $d$-chloroform) of the tethered monomer 8 synthesized in 2-ethoxyethanol. The signal at $\delta 7.30-7.35$ indicates the presence of hydrodebrominated derivatives, generated as byproducts during the complexation reaction in 2-ethoxyethanol.

The tethered monomer 8 was successfully synthesized in acetonitrile at $80{ }^{\circ} \mathrm{C}$ :

A mixture of 13-(2,7-dibromo-9-octyl-9H-fluoren-9-yl)tridecane-2,4-dione 6 (242 mg, $374 \mu \mathrm{mol})$, crude di- $\mu$-chloro- $N, N^{\prime}$-trans- $N^{\prime \prime}, N^{\prime \prime \prime}$-trans-tetrakis[2-(2-pyridyl- $\left.\kappa N\right)$ benzo[b]thien-3-yl]diiridium(III) 7 (194 $\mathrm{mg}$, ca. $0.15 \mathrm{mmol})$ and sodium carbonate $(159 \mathrm{mg}, 1.50 \mathrm{mmol})$ in acetonitrile $\left(10 \mathrm{~cm}^{3}\right)$ was stirred for $48 \mathrm{~h}$ at $80{ }^{\circ} \mathrm{C}$. The mixture was cooled to $\mathrm{RT}$ and the solvent was evaporated under reduced pressure. The crude mixture was purified by dry flash chromatography using hexane-ethyl acetate (1:0 changing to 9:1) as eluent. The pure product was taken up in DCM-methanol and the solvents were evaporated under reduced pressure to leave the tethered monomer $\mathbf{8}(280 \mathrm{mg}, \mathrm{ca} .74 \%)$ as a red powder.

Anal. Calcd for $\mathrm{C}_{60} \mathrm{H}_{61} \mathrm{Br}_{2} \mathrm{IrN}_{2} \mathrm{O}_{2} \mathrm{~S}_{2}$ : C, 57.3; H, 4.9; N, 2.2. Found: C, 57.0; H, 4.9; N, 2.3. $R_{\mathrm{f}}(\mathrm{DCM})$ 0.8. UV $\left(\mathrm{CHCl}_{3}\right) \lambda_{\max }(\log \varepsilon): 284$ (4.8), 316 (4.6), 340 (4.4), 360 (sh, 4.3), 430 (sh, 3.5), 464 (sh, 3.8), $490 \mathrm{~nm}$ (3.9). IR $\left(\mathrm{CHCl}_{3}\right) v_{\max }: 2930,2856,1577,1477 \mathrm{~cm}^{-1} .{ }^{1} \mathrm{H}$ NMR $\left(400 \mathrm{MHz}, \mathrm{CDCl}_{3}, \delta\right): 0.53-0.64$ $\left(\mathrm{m}, 4 \mathrm{H}, 2 \times \mathrm{CH}_{2}\right), 0.83\left(\mathrm{t}, J=7.1 \mathrm{~Hz}, 3 \mathrm{H}, \mathrm{CH}_{3}\right), 0.74-1.28\left(\mathrm{~m}, 22 \mathrm{H}, 11 \times \mathrm{CH}_{2}\right), 1.77(\mathrm{~s}, 3 \mathrm{H}$, tridecyl 1- $\left.\mathrm{H}_{3}\right), 1.87-1.97\left(\mathrm{~m}, 6 \mathrm{H}\right.$, tridecyl 5- $\mathrm{H}_{2}$ and $\left.2 \times \mathrm{CH}_{2}\right), 5.21(\mathrm{~s}, 1 \mathrm{H}$, tridecyl 3-H), $6.20(\mathrm{~d}, J=7.9 \mathrm{~Hz}, 1$ 
H, benzo 4-H or 7-H), $6.27(\mathrm{~d}, J=8.1 \mathrm{~Hz}, 1 \mathrm{H}$, benzo 4-H or 7-H), 6.76-6.84 (m, $2 \mathrm{H}, 2 \times$ benzo 5-H or 6-H), 6.90-6.99 (m, 2 H, $2 \times$ pyridyl 5-H), 7.01-7.08 (m, 2 H, $2 \times$ benzo 5-H or 6-H), 7.42-7.48 (m, 4 $\mathrm{H}, 4 \times \mathrm{ArH}), 7.49-7.55(\mathrm{~m}, 3 \mathrm{H}$, pyridyl-A 3-H and $2 \times \mathrm{ArH}), 7.60-7.67(\mathrm{~m}, 4 \mathrm{H}$, pyridyl-A 4-H, pyridyl-B 3-H and $2 \times$ benzo 4-H or 7-H), 7.75 (dt, $J=1.5 \mathrm{~Hz}$ and $7.7 \mathrm{~Hz}, 1 \mathrm{H}$, pyridyl-B 4-H), 8.40 $\left(\mathrm{d}, J=5.6 \mathrm{~Hz}, 2 \mathrm{H}, 2 \times\right.$ pyridyl 6-H). ${ }^{13} \mathrm{C} \mathrm{NMR}\left(\mathrm{CDCl}_{3}, \delta\right): 14.0(\mathrm{q}), 22.5(\mathrm{t}), 23.6(\mathrm{t}), 23.7(\mathrm{t}), 27.0(\mathrm{t})$, $28.5(\mathrm{q}), 28.6(\mathrm{t}), 29.1(\mathrm{t}), 29.2(\mathrm{t}), 29.3(\mathrm{t}), 29.8(\mathrm{t}), 29.8(\mathrm{t}), 31.7(\mathrm{t}), 40.2(\mathrm{t}), 41.2(\mathrm{t}), 55.7(\mathrm{~s}), 100.1$ (d), 118.0 (d), 118.1 (d), 118.3 (d), 118.5 (d), 121.2 (d), 121.5 (s), 122.6 (d), 123.5 (d), 124.7 (d), 124.8 (d), 125.6 (d), 125.8 (d), 126.1 (d), 130.2 (d), 134.9 (s), 135.0 (s), 137.8 (d), 137.9 (d), 139.1 (s), 142.2 (s), 142.3 (s), 146.4 (s), 146.7 (s), 146.8 (s), 146.9 (s), 149.1 (d), 149.2 (d), 152.5 (s), 165.9 (s), 166.1 (s), 184.5 (s), 188.3 (s). MS-MALDI m/z (\% relative intensity): 1260 (56), 1258 (100), 1256 (79), 1254 (19).

A diminished yield of the tethered monomer $8(187 \mathrm{mg}, c a .50 \%)$ was obtained in an identical experiment where the reaction was stopped after $24 \mathrm{~h}$ at $80^{\circ} \mathrm{C}$. 

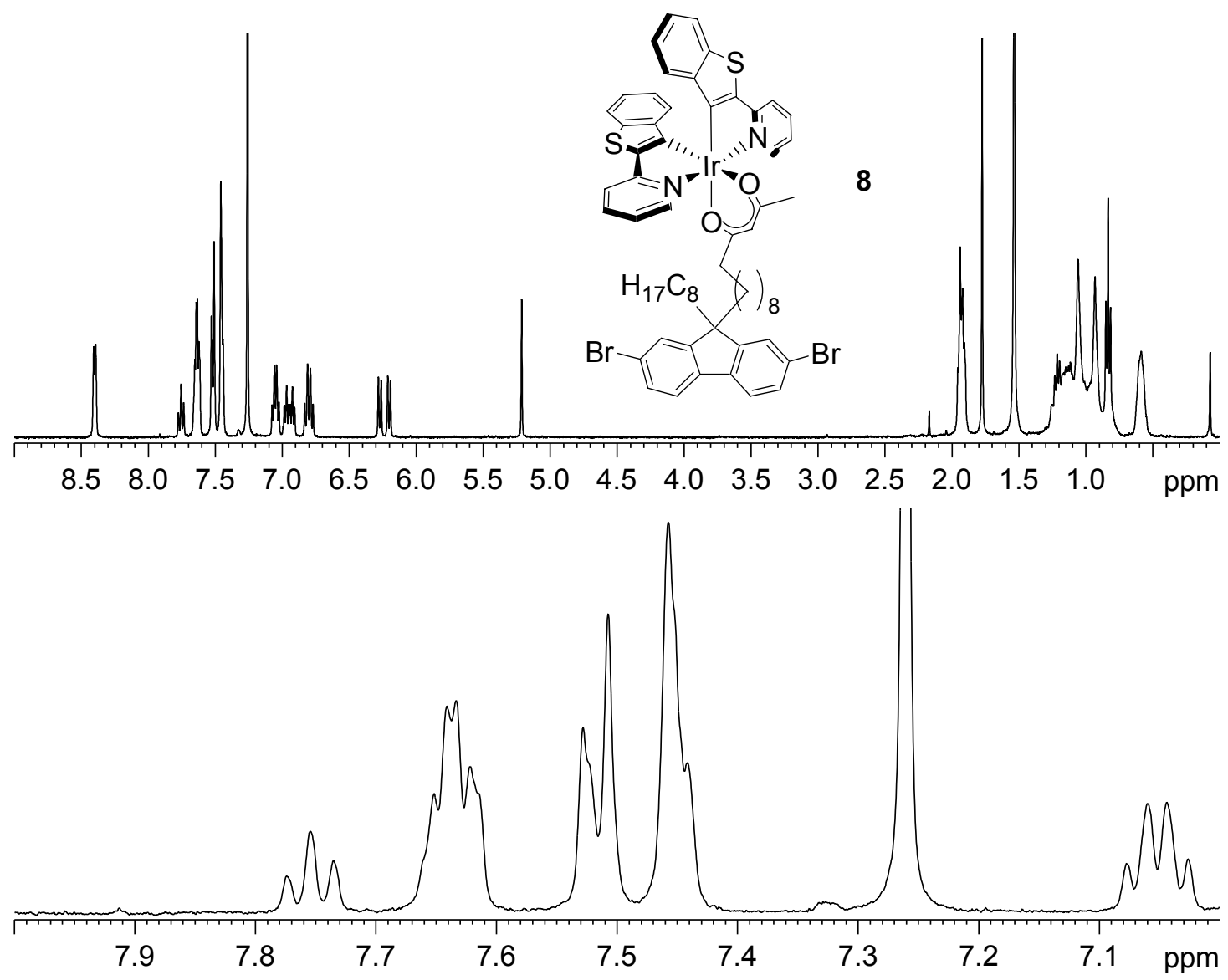

Figure S2. (a) Proton NMR spectrum (400 MHz, $d$-chloroform) of the tethered monomer 8 synthesized in acetonitrile. (b) Detail from the aromatic region.

\section{6-Bromomethyl-4-methoxy-2H-pyran-2-one $9^{7}$}<smiles>COc1cc(CBr)oc(=O)c1</smiles>

A mixture of 4-methoxy-6-methyl-2H-pyran-2-one (500 mg, $3.57 \mathrm{mmol}), \mathrm{N}$-bromosuccinimide (700 $\mathrm{mg}, 3.93 \mathrm{mmol}$ ) and 2,2'-azobis(2-methylpropionitrile) $(7 \mathrm{mg}, 0.04 \mathrm{mmol})$ in tetrachloromethane (125 $\mathrm{cm}^{3}$ ) was stirred for $1.5 \mathrm{~h}$ under reflux and illumination from a $250 \mathrm{~W}$ floodlamp. The mixture was cooled to RT and the solvents were evaporated under reduced pressure. The crude mixture was filtered through a pad of silica using hexane-ethyl acetate $(1: 1)$ as eluent to give the crude bromopyrone 9 (779 mg, ca. 63 wt.\% by proton NMR, ca. $63 \%$ yield) as a pale yellow solid. 
${ }^{1} \mathrm{H}$ NMR $\left(400 \mathrm{MHz}, \mathrm{CDCl}_{3}, \delta\right): 3.82\left(\mathrm{~s}, 3 \mathrm{H}, \mathrm{OCH}_{3}\right), 4.10\left(\mathrm{~s}, 2 \mathrm{H}, \mathrm{CH}_{2}\right), 5.49$ (d, J=2.2 Hz, $1 \mathrm{H}, 3-$ $\mathrm{H}), 6.08(\mathrm{~d}, J=2.2 \mathrm{~Hz}, 1 \mathrm{H}, 5-\mathrm{H})$. The selected ${ }^{1} \mathrm{H}$ NMR data are in agreement with literature values for the pure bromopyrone $9 .^{7}$

\section{4-Methoxy-6-[(2,7-dibromo-9-octyl-9H-fluoren-9-yl)methyl]-2H-pyran-2-one 10}

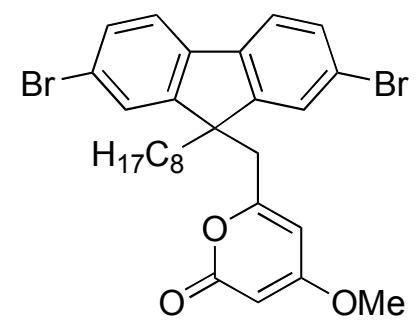

Butyllithium (1.6 M in hexane; $\left.2.5 \mathrm{~cm}^{3}, 4.0 \mathrm{mmol}\right)$ was added dropwise over $10 \mathrm{~min}$ to a stirred solution of diisopropylamine $\left(0.60 \mathrm{~cm}^{3}, 4.3 \mathrm{mmol}\right)$ in $\mathrm{THF}\left(10 \mathrm{~cm}^{3}\right)$ at $0{ }^{\circ} \mathrm{C}$. The solution was stirred for $30 \mathrm{~min}$ at $0{ }^{\circ} \mathrm{C}$ before cooling to $-78{ }^{\circ} \mathrm{C}$. A solution of 2,7-dibromo-9-octyl-9H-fluorene 3 (1.74 g, 4.00 mmol) in THF $\left(7 \mathrm{~cm}^{3}\right)$ was added dropwise over $10 \mathrm{~min}$ and the resultant red solution was stirred for $1.25 \mathrm{~h}$ at $-78^{\circ} \mathrm{C}$. A solution of crude 6-bromomethyl-4-methoxy-2H-pyran-2-one 9 (695 mg, ca. 2.0 mmol) in THF $\left(5 \mathrm{~cm}^{3}\right)$ was added dropwise over 15 min at $-78^{\circ} \mathrm{C}$. The resultant bright red mixture was stirred for $30 \mathrm{~min}$ at $-78{ }^{\circ} \mathrm{C}$ before it was allowed to warm to $\mathrm{RT}$ and the resultant dark red solution was stirred overnight. The solvents were evaporated under reduced pressure and the crude mixture was purified by dry flash chromatography using hexane-ethyl acetate (1:0 changing to $3: 1)$ as eluent. The crude product was purified by Kugelrohr distillation $\left(250{ }^{\circ} \mathrm{C}, 0.1 \mathrm{mmHg}\right)$ and dry flash chromatography using hexane-ethyl acetate (1:0 changing to 1:1) as eluent to give the pyrone $\mathbf{1 0}(996 \mathrm{mg}, \mathrm{ca} .87 \%, 55 \%$ over two steps from 4-methoxy-6-methyl-2H-pyran-2-one) as a pale yellow glass.

Anal. Calcd for $\mathrm{C}_{28} \mathrm{H}_{30} \mathrm{Br}_{2} \mathrm{O}_{3}: \mathrm{C}, 58.6 ; \mathrm{H}, 5.3$. Found: C, 58.5; H, 5.2. $R_{\mathrm{f}}$ (hexane-ethyl acetate, 1:1) 0.4. IR $\left(\mathrm{CHCl}_{3}\right) v_{\text {max }}: 2930,2857,1711 \mathrm{br} \mathrm{cm}^{-1} .{ }^{1} \mathrm{H} \mathrm{NMR}\left(500 \mathrm{MHz}, \mathrm{CDCl}_{3}, \delta\right): 0.54-0.63(\mathrm{~m}, 2 \mathrm{H}$, $\left.\mathrm{CH}_{2}\right), 0.83\left(\mathrm{t}, J=7.3 \mathrm{~Hz}, 3 \mathrm{H}, \mathrm{CH}_{3}\right), 1.00-1.26\left(\mathrm{~m}, 10 \mathrm{H}, 5 \times \mathrm{CH}_{2}\right), 2.03-2.09\left(\mathrm{~m}, 2 \mathrm{H}, \mathrm{CH}_{2}\right), 2.96(\mathrm{~s}, 2$ $\left.\mathrm{H}, \mathrm{CH}_{2}\right), 3.65\left(\mathrm{~s}, 3 \mathrm{H}, \mathrm{OCH}_{3}\right), 5.12(\mathrm{~d}, J=2.1 \mathrm{~Hz}, 1 \mathrm{H}$, pyranyl 3-H or 5-H), $5.25(\mathrm{~d}, J=2.1 \mathrm{~Hz}, 1 \mathrm{H}$, 
pyranyl 3-H or 5-H), 7.45-7.49 (m, $4 \mathrm{H}, 4 \times \mathrm{ArH}), 7.50(\mathrm{~d}, J=7.9 \mathrm{~Hz}, 2 \mathrm{H}, 2 \times \mathrm{ArH}) .{ }^{13} \mathrm{C} \mathrm{NMR}$ $\left(\mathrm{CDCl}_{3}, \delta\right): 14.0(\mathrm{q}), 22.6(\mathrm{t}), 23.5(\mathrm{t}), 29.1(\mathrm{t}), 29.1(\mathrm{t}), 29.6(\mathrm{t}), 31.7(\mathrm{t}), 38.9(\mathrm{t}), 43.2(\mathrm{t}), 54.5(\mathrm{~s}), 55.7$

(q), 88.0 (d), 101.8 (d), 121.4 (d), 121.6 (s), 126.7 (d), 131.0 (d), 138.6 (s), 150.1 (s), 160.9 (s), 164.0

(s), 170.3 (s). HRMS-ES $(m / z):[\mathrm{M}+\mathrm{H}]^{+}$calcd for $\mathrm{C}_{28} \mathrm{H}_{31}{ }^{79} \mathrm{Br}_{2} \mathrm{O}_{3}$, 573.0634; found, 573.0634. MS-EI $m / z$ (\% relative intensity): $575(15), 323(50), 189(31), 176(67), 140$ (100), 125 (49), 69 (65), 57 (98).

\section{1-(2,7-Dibromo-9-octyl-9H-fluoren-9-yl)pentane-2,4-dione 11}

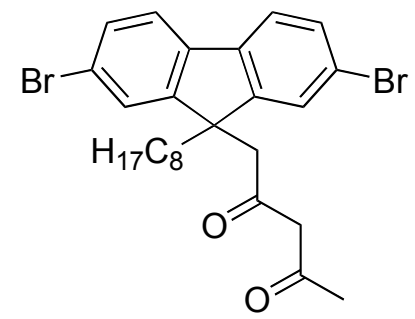

Hydrochloric acid $\left(1 \mathrm{M} ; 3 \mathrm{~cm}^{3}, 3 \mathrm{mmol}\right)$ was added to a stirred solution of 4-methoxy-6-[(2,7dibromo-9-octyl-9H-fluoren-9-yl)methyl]-2H-pyran-2-one $10(813 \mathrm{mg}, 1.95 \mathrm{mmol})$ in glacial acetic acid $\left(30 \mathrm{~cm}^{3}\right)$ at RT and the mixture was stirred for $1 \mathrm{~d}$ at $100^{\circ} \mathrm{C}$. The mixture was cooled to RT and the solvents were evaporated under reduced pressure. The conversion of the pyrone $\mathbf{1 0}$ to the dione $\mathbf{1 1}$ was estimated to be $69 \%$ by proton NMR from the ratio of the integral of the enolone tautomer $3-\mathrm{H}(\delta 5.01)$ of the dione 11 to the integral of the reaction mixture aromatic protons $(\delta 7.44-7.56)$. The crude mixture was filtered through a pad of silica using DCM as eluent to give the crude dione 11 (745 mg, $c a .86$ wt.\% 11) as an orange solid that was used without further purification.

The dione $\mathbf{1 1}$ was isolated in a pure form through decomplexation:

p-Toluenesulfonic acid monohydrate $(12 \mathrm{mg}, 63 \mu \mathrm{mol})$ was added to a stirred solution of [1-(2,7dibromo-9-octyl-9H-fluoren-9-yl)pentane-2,4-dionato]- $N, N^{\prime}$-trans-bis[2-(2-pyridyl- $\left.\kappa N\right)$ benzo[b]thien-3yl]iridium(III) $12(50 \mathrm{mg}, 44 \mu \mathrm{mol})$ in DCM-acetonitrile $\left(1: 1 ; 20 \mathrm{~cm}^{3}\right)$ at RT and the solution was stirred for $30 \mathrm{~min}$. Additional $p$-toluenesulfonic acid monohydrate $(8 \mathrm{mg}, 0.04 \mathrm{mmol})$ was added and 
the solution was stirred for $1.5 \mathrm{~h}$, after which time the spacerless monomer 12 was no longer visible by analytical TLC. The solvent was evaporated under reduced pressure and the crude mixture was filtered through a pad of silica using hexane-DCM (1:3) as eluent to give the dione 11 (23 mg, quantitative yield) as an orange oil that eventually crystallized to an orange solid, mp $76-78{ }^{\circ} \mathrm{C}$.

Anal. Calcd for $\mathrm{C}_{26} \mathrm{H}_{30} \mathrm{Br}_{2} \mathrm{O}_{2}$ : C, 58.4; H, 5.7. Found: C, 58.5; H, 5.7. $R_{\mathrm{f}}(\mathrm{DCM})$ 0.6. IR $\left(\mathrm{CHCl}_{3}\right) v_{\max }$ : 2930, 2857, 1600 $\mathrm{br} \mathrm{cm}^{-1} .{ }^{1} \mathrm{H}$ NMR (500 MHz, $\left.\mathrm{CDCl}_{3}, \delta\right): 0.52-0.64\left(\mathrm{~m}, 2 \mathrm{H}, \mathrm{CH}_{2}\right), 0.83(\mathrm{t}, J=7.2 \mathrm{~Hz}$, $\left.3 \mathrm{H}, \mathrm{CH}_{3}\right), 1.00-1.31\left(\mathrm{~m}, 10 \mathrm{H}, 5 \times \mathrm{CH}_{2}\right), 1.90\left(\mathrm{~s}, 2.7 \mathrm{H}\right.$, enolone 5- $\left.\mathrm{H}_{3}\right), 1.94\left(\mathrm{~s}, 0.3 \mathrm{H}\right.$, dione 5- $\left.\mathrm{H}_{3}\right)$, 1.96-2.01 (m, $0.2 \mathrm{H}, \mathrm{CH}_{2}$ (dione)), 2.01-2.08 (m, 1.8 H, $\mathrm{CH}_{2}$ (enolone)), 2.76 (s, $1.8 \mathrm{H}$, enolone 1- $\mathrm{H}_{2}$ ), $3.09\left(\mathrm{~s}, 0.2 \mathrm{H}\right.$, dione $\left.3-\mathrm{H}_{2}\right), 3.16\left(\mathrm{~s}, 0.2 \mathrm{H}\right.$, dione 1- $\left.\mathrm{H}_{2}\right), 5.01(\mathrm{~s}, 0.9 \mathrm{H}$, enolone 3-H), $7.48(\mathrm{dd}, J=1.7$ $\mathrm{Hz}$ and $8.0 \mathrm{~Hz}, 1.8 \mathrm{H}, 2 \times \mathrm{ArH}($ enolone)), 7.49-7.57 (m, 4.2 H, $2 \times \mathrm{ArH}$ (dione) and $4 \times \mathrm{ArH}), 15.07$ (br s, $0.9 \mathrm{H}$, enolone $\mathrm{OH}) .{ }^{13} \mathrm{C} \mathrm{NMR}\left(\mathrm{CDCl}_{3}, \delta\right): 14.0(\mathrm{q}), 22.6(\mathrm{t}), 23.2(\mathrm{t}), 23.5(\mathrm{t}), 25.2(\mathrm{q}), 29.1(\mathrm{t})$, $29.1(\mathrm{t}), 29.6(\mathrm{t}), 29.6(\mathrm{t}), 29.7(\mathrm{t}), 30.5(\mathrm{q}), 31.7(\mathrm{t}), 39.0(\mathrm{t}), 39.5(\mathrm{t}), 47.3(\mathrm{t}), 51.7(\mathrm{t}), 52.8(\mathrm{~s}), 53.9(\mathrm{~s})$, 58.5 (t), 101.7 (d), 121.3 (d), 121.3 (s), 121.4 (d), 121.7 (s), 126.5 (d), 130.7 (d), 130.9 (d), 138.6 (s), 138.7 (s), 150.8 (s), 150.9 (s), 188.4 (s), 192.3 (s), 200.5 (s), 201.8 (s). HRMS-ES (m/z): [M + $\left.\mathrm{NH}_{4}\right]^{+}$ calcd for $\mathrm{C}_{26} \mathrm{H}_{34}{ }^{79} \mathrm{Br}_{2} \mathrm{NO}_{2}, 550.0951$; found, 550.0947. MS-EI $m / z$ (\% relative intensity): 534 (8), 323 (9), 189 (10), $176(20), 85$ (62), 57 (35), 43 (100). 


\section{[1-(2,7-Dibromo-9-octyl-9H-fluoren-9-yl)pentane-2,4-dionato]- $N$, $N^{\prime}$-trans-bis [2-(2-pyridyl- $\left.\kappa \mathrm{N}\right)-$}

\section{benzo[b]thien-3-yl]iridium(III) 12}

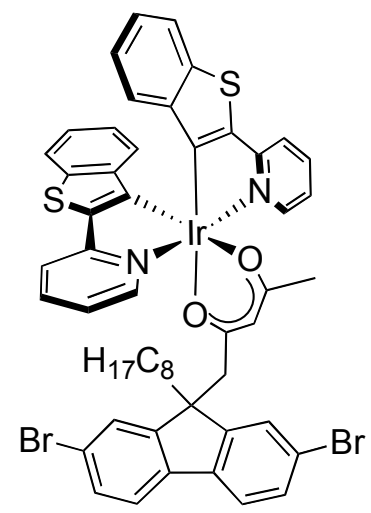

A mixture of crude di- $\mu$-chloro- $N, N^{\prime}$-trans- $N^{\prime \prime}, N^{\prime \prime \prime}$-trans-tetrakis[2-(2-pyridyl- $\left.\kappa N\right)$ benzo[b]thien-3-yl]diiridium(III) 7 (194 mg, ca. $0.15 \mathrm{mmol}$ ), crude 1-(2,7-dibromo-9-octyl-9H-fluoren-9-yl)pentane-2,4-dione $11(250 \mathrm{mg}, c a .0 .40 \mathrm{mmol})$ and sodium carbonate $(159 \mathrm{mg}, 1.50 \mathrm{mmol})$ in acetonitrile $\left(10 \mathrm{~cm}^{3}\right)$ was stirred for $48 \mathrm{~h}$ at $80{ }^{\circ} \mathrm{C}$. The mixture was cooled to RT and the solvent was evaporated under reduced pressure. The crude mixture was purified by dry flash chromatography using hexane-ethyl acetate (1:0 changing to $24: 1)$ as eluent for the impurities and hexane-DCM (7:3 changing to $1: 1)$ as eluent for the product. The pure product was taken up in DCM-methanol and the solvents were evaporated under reduced pressure to leave the spacerless monomer $12(278 \mathrm{mg}, \mathrm{ca}$. 81\%) as an orange powder.

Anal. Calcd for $\mathrm{C}_{52} \mathrm{H}_{45} \mathrm{Br}_{2} \mathrm{IrN}_{2} \mathrm{O}_{2} \mathrm{~S}_{2}$ : C, 54.5; H, 4.0; N, 2.4. Found: C, 54.5; H, 4.0; N, 2.3. $R_{\mathrm{f}}(\mathrm{DCM})$ 0.6. UV $\left(\mathrm{CHCl}_{3}\right) \lambda_{\max }(\log \varepsilon): 284$ (4.7), 316 (4.5), 342 (4.3), 360 (sh, 4.2), 430 (sh, 3.4), 464 (sh, 3.7), $492 \mathrm{~nm}$ (3.8). IR $\left(\mathrm{CHCl}_{3}\right) v_{\max }: 2930,2855,1602,1477 \mathrm{~cm}^{-1} .{ }^{1} \mathrm{H}$ NMR (500 MHz, $\left.\mathrm{CDCl}_{3}, \delta\right): 0.38-0.46$ (m, $\left.2 \mathrm{H}, \mathrm{CH}_{2}\right), 0.80\left(\mathrm{t}, J=7.2 \mathrm{~Hz}, 3 \mathrm{H}, \mathrm{CH}_{3}\right), 0.86-1.21\left(\mathrm{~m}, 10 \mathrm{H}, 5 \times \mathrm{CH}_{2}\right), 1.55-1.60\left(\mathrm{~m}, 2 \mathrm{H}, \mathrm{CH}_{2}\right)$, $1.66\left(\mathrm{~s}, 3 \mathrm{H}\right.$, pentyl 5- $\left.\mathrm{H}_{3}\right), 2.87\left(\mathrm{~d}, J=17.6 \mathrm{~Hz}, 1 \mathrm{H}\right.$, pentyl 1- $\left.\mathrm{H}_{\text {gem }}\right), 3.00(\mathrm{~d}, J=17.6 \mathrm{~Hz}, 1 \mathrm{H}$, pentyl 1$\left.\mathrm{H}_{\text {gem }}\right), 5.13(\mathrm{~s}, 1 \mathrm{H}$, pentyl 3-H), $5.82(\mathrm{~d}, J=8.0 \mathrm{~Hz}, 1 \mathrm{H}$, benzo-A 4-H or 7-H), $6.03(\mathrm{~d}, J=8.1 \mathrm{~Hz}, 1 \mathrm{H}$, benzo-B 4-H or 7-H), 6.36 (d, $J=8.0 \mathrm{~Hz}, 1 \mathrm{H}$, fluorenyl 4-H), 6.62 (d, $J=8.0 \mathrm{~Hz}, 1 \mathrm{H}$, fluorenyl 5-H), 6.69-6.74 (m, $1 \mathrm{H}$, benzo-B 5-H or 6-H), 6.80-6.84 (m, 2 H, benzo-A 5-H or 6-H and fluorenyl 3-H), 
6.89-6.92 (m, $1 \mathrm{H}$, pyridyl-C 5-H), $6.93(\mathrm{~d}, J=1.8 \mathrm{~Hz}, 1 \mathrm{H}$, fluorenyl 8-H), 6.95-7.01 (m, $2 \mathrm{H}$, pyridyl-D 5-H and benzo-B 5-H or 6-H), 7.12 (dd, $J=1.8 \mathrm{~Hz}$ and 8.0 Hz, $1 \mathrm{H}$, fluorenyl 6-H), 7.13$7.17(\mathrm{~m}, 1 \mathrm{H}$, benzo-A 5-H or 6-H), $7.20(\mathrm{~d}, J=5.4 \mathrm{~Hz}, 1 \mathrm{H}$, pyridyl-D 6-H), $7.36(\mathrm{~d}, J=1.7 \mathrm{~Hz}, 1 \mathrm{H}$, fluorenyl 1-H), 7.41 (d, $J=8.0 \mathrm{~Hz}, 1 \mathrm{H}$, pyridyl-C 3-H), 7.45 (d, $J=7.9 \mathrm{~Hz}, 1 \mathrm{H}$, pyridyl-D 3-H), 7.54 (d, $J=7.9 \mathrm{~Hz}, 1 \mathrm{H}$, benzo-B 4-H or 7-H), 7.67 (dt, $J=1.4 \mathrm{~Hz}$ and 7.9 Hz, $1 \mathrm{H}$, pyridyl-D 4-H), 7.707.75 (m, 2 H, pyridyl-C 4-H and benzo-A 4-H or 7-H), $8.13(\mathrm{~d}, J=5.6 \mathrm{~Hz}, 1 \mathrm{H}$, pyridyl-C $6-\mathrm{H}) .{ }^{13} \mathrm{C}$ $\operatorname{NMR}\left(\mathrm{CDCl}_{3}, \delta\right): 14.0(\mathrm{q}), 22.5(\mathrm{t}), 22.8(\mathrm{t}), 28.3(\mathrm{q}), 29.1(\mathrm{t}), 29.1(\mathrm{t}), 29.6(\mathrm{t}), 31.7(\mathrm{t}), 41.2(\mathrm{t}), 48.1$ (t), 51.9 (s), 99.6 (d), 117.9 (d), 118.1 (d), 118.3 (d), 118.9 (d), 119.9 (s), 120.3 (s), 121.0 (d), 121.4 (d), 122.2 (d), 122.5 (d), 123.1 (d), 123.4 (d), 123.7 (d), 124.5 (d), 124.6 (d), 124.6 (d), 125.4 (d), 125.9 (d), 129.6 (d), 129.9 (d), 134.3 (s), 134.8 (s), 137.6 (d), 137.7 (d), 138.5 (s), 139.5 (s), 142.0 (s), 142.2 (s), 144.0 (s), 144.7 (s), 146.5 (s), 146.8 (s), 148.5 (d), 149.1 (d), 152.2 (s), 152.4 (s), 165.5 (s), 165.5 (s), 182.4 (s), 183.8 (s). MS-MALDI m/z (\% relative intensity): 1150 (14), 1148 (57), 1146 (100), 1144 (76), 1142 (23), 714 (12), $712(96), 710(38)$.

A diminished yield of the spacerless monomer $12(181 \mathrm{mg}, \mathrm{ca} .53 \%)$ was obtained in an identical experiment where the reaction was stopped after $24 \mathrm{~h}$ at $80^{\circ} \mathrm{C}$. 

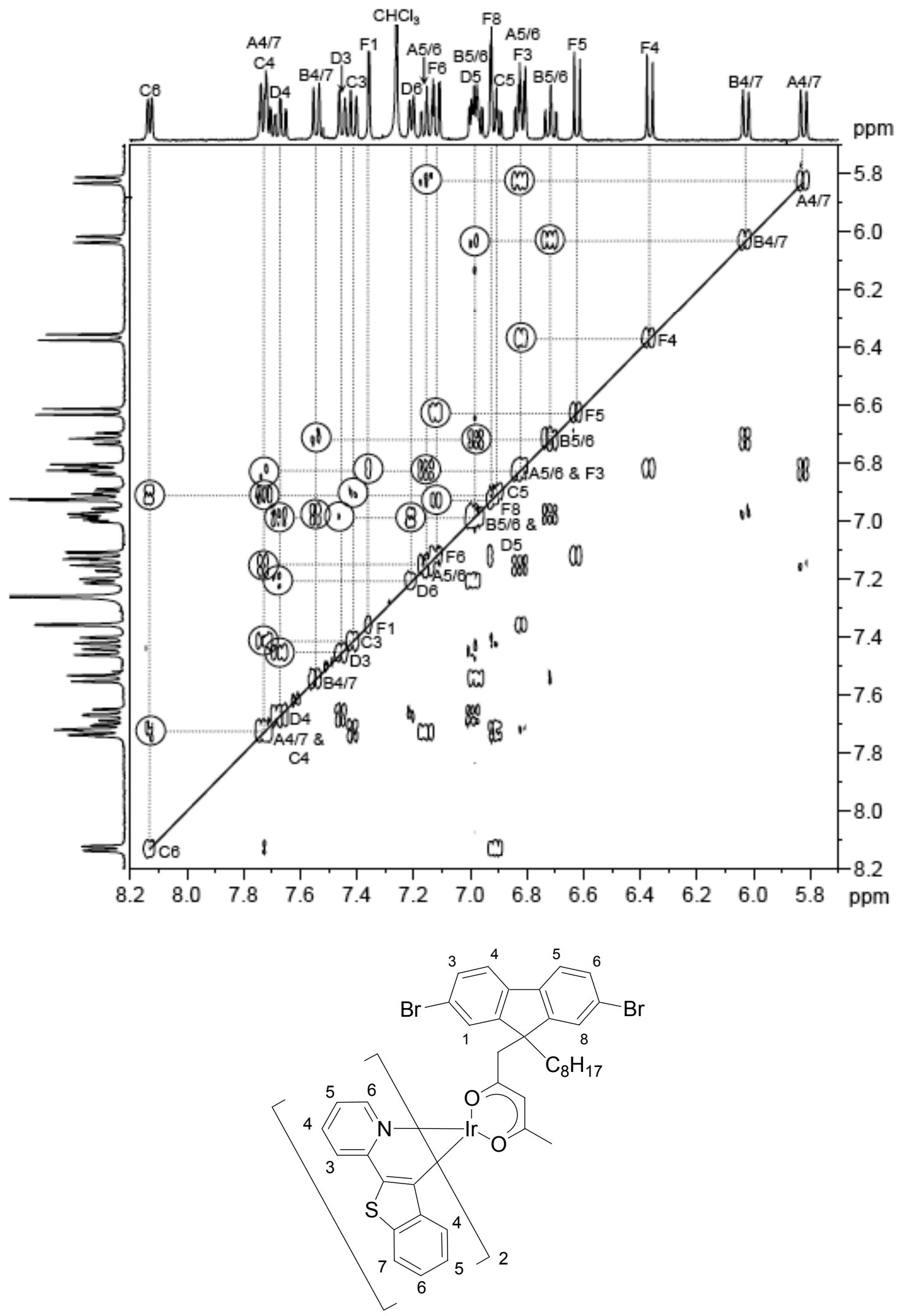

Figure S3. The aromatic region from a double-quantum filtered COSY spectrum (400 MHz, $d$ chloroform) of the spacerless monomer 12. A and B denote benzo[b]thienyl, $\mathrm{C}$ and $\mathrm{D}$ denote pyridyl and $\mathrm{F}$ denotes the fluorenyl ring system. 


\section{(Pentane-2,4-dionato)- $N, N^{\prime}$-trans-bis[2-(2-pyridyl- $\left.\kappa N\right)$ benzo[b]thien-3-yl]iridium(III) 13}

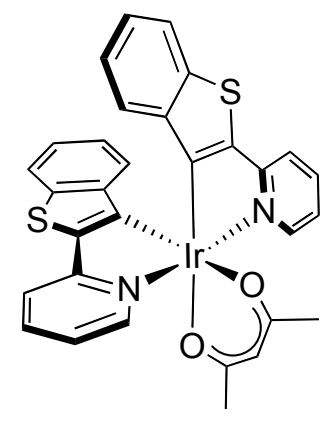

A mixture of crude di- $\mu$-chloro- $N, N^{\prime}$-trans- $N^{\prime \prime}, N^{\prime \prime \prime}$-trans-tetrakis[2-(2-pyridyl- $\left.\kappa N\right)$ benzo[b]thien-3-yl]diiridium(III) 7 (45 mg, ca. $35 \mu \mathrm{mol})$, pentane-2,4-dione 5 (10 mg, $0.10 \mathrm{mmol})$ and sodium carbonate (37 mg, $0.35 \mathrm{mmol})$ in 2-ethoxyethanol $\left(2 \mathrm{~cm}^{3}\right)$ was stirred for $18 \mathrm{~h}$ under reflux. The mixture was cooled to RT, diluted with water $\left(8 \mathrm{~cm}^{3}\right)$ and the resultant precipitate was isolated by filtration and washed thoroughly with water. 2-(Benzo[b]thien-2-yl)pyridine was sublimed from the mixture $\left(120{ }^{\circ} \mathrm{C}\right.$, $0.2 \mathrm{mmHg})$ before the crude product was purified by dry flash chromatography using hexane-DCM (1:0 changing to $6: 1$ ) as eluent. The pure product was taken up in hexane-DCM and the solvents were evaporated under reduced pressure to leave the complex 13 (36 mg, ca. 73\%) as a red powder.

Anal. Calcd for $\mathrm{C}_{31} \mathrm{H}_{23} \mathrm{IrN}_{2} \mathrm{O}_{2} \mathrm{~S}_{2}$ : C, 52.3; H, 3.3; N, 3.9. Found: C, 52.0; H, 3.2; N, 3.7. $R_{\mathrm{f}}(\mathrm{DCM})$ 0.6. UV $\left(\mathrm{CHCl}_{3}\right) \lambda_{\max }(\log \varepsilon): 288$ (4.5), $328(\mathrm{sh}, 4.3), 340$ (4.3), 362 (sh, 4.2), 432 (sh, 3.5), 464 (sh, 3.7), $490 \mathrm{~nm}$ (3.8). IR $\left(\mathrm{CHCl}_{3}\right) v_{\max }: 1603,1580 \mathrm{~cm}^{-1} .{ }^{1} \mathrm{H} \mathrm{NMR}\left(500 \mathrm{MHz}, \mathrm{CDCl}_{3}, \delta\right): 1.78(\mathrm{~s}, 6 \mathrm{H}$, pentyl 1- $\mathrm{H}_{3}$ and 5- $\left.\mathrm{H}_{3}\right), 5.26(\mathrm{~s}, 1 \mathrm{H}$, pentyl 3-H), $6.20(\mathrm{~d}, J=8.1 \mathrm{~Hz}, 2 \mathrm{H}, 2 \times$ benzo 4-H or 7-H), 6.80 $(\mathrm{t}, J=7.3 \mathrm{~Hz}, 2 \mathrm{H}, 2 \times$ benzo $5-\mathrm{H}$ or $6-\mathrm{H}), 7.00(\mathrm{t}, J=6.0 \mathrm{~Hz}, 2 \mathrm{H}, 2 \times$ pyridyl $5-\mathrm{H}), 7.05(\mathrm{t}, J=7.3 \mathrm{~Hz}$, $2 \mathrm{H}, 2 \times$ benzo 5-H or 6-H), 7.62-7.65 (m, $4 \mathrm{H}, 4 \times \mathrm{ArH}), 7.77(\mathrm{t}, J=7.7 \mathrm{~Hz}, 2 \mathrm{H}, 2 \times \mathrm{ArH}), 8.44(\mathrm{~d}, J$ $=6.0 \mathrm{~Hz}, 2 \mathrm{H}, 2 \times$ pyridyl 6-H). ${ }^{1} \mathrm{H}$ NMR $\left(250 \mathrm{MHz}, d_{6}\right.$-acetone, $\left.\delta\right): 1.72\left(\mathrm{~s}, 6 \mathrm{H}\right.$, pentyl 1- $\mathrm{H}_{3}$ and $\left.5-\mathrm{H}_{3}\right)$, $5.37(\mathrm{~s}, 1 \mathrm{H}$, pentyl 3-H), $6.24(\mathrm{~d}, J=8.1 \mathrm{~Hz}, 2 \mathrm{H}, 2 \times \mathrm{ArH}), 6.74-6.83(\mathrm{~m}, 2 \mathrm{H}, 2 \times \mathrm{ArH}), 7.01-7.10$ (m, 2 H, $2 \times A r H), 7.19-7.27(\mathrm{~m}, 2 \mathrm{H}, 2 \times \mathrm{ArH}), 7.64-7.77(\mathrm{~m}, 4 \mathrm{H}, 4 \times \mathrm{ArH}), 7.93-8.02(\mathrm{~m}, 2 \mathrm{H}, 2 \times$ ArH), 8.54 (d, $J=8.5 \mathrm{~Hz}, 2 \mathrm{H}, 2 \times$ pyridyl 6-H). ${ }^{13} \mathrm{C} \mathrm{NMR}\left(\mathrm{CDCl}_{3}, \delta\right): 28.4$ (q), 100.5 (d), 118.2 (d), 118.6 (d), 122.7 (d), 123.5 (d), 124.8 (d), 125.6 (d), 135.0 (s), 138.0 (d), 142.2 (s), 145.9 (s), 146.8 (s), 
149.1 (d), 166.1 (s), 184.6 (s). HRMS-ES $(m / z):[\mathrm{M}+\mathrm{H}]^{+}$calcd for $\mathrm{C}_{31} \mathrm{H}_{24} \mathrm{IrN}_{2} \mathrm{O}_{2} \mathrm{~S}_{2}, 713.0903$; found, 713.0906. MS-EI m/z (\% relative intensity): 714 (12), 713 (21), 712 (74), 710 (36), 615 (17), 614 (26), 613 (100), 611 (59), $342(57), 286$ (83). Although the ${ }^{1} \mathrm{H}$ NMR ( $d_{6}$-acetone) data reported here are in some disagreement with literature values, ${ }^{2}$ the additional characterization is consistent with the expected product.

The complex 13 was also prepared in acetonitrile at $80^{\circ} \mathrm{C}$ :

A mixture of crude di- $\mu$-chloro- $N, N^{\prime}$-trans- $N^{\prime \prime}, N^{\prime \prime \prime}$-trans-tetrakis[2-(2-pyridyl- $\left.\kappa N\right)$ benzo[b]thien-3-yl]diiridium(III) 7 (194 mg, ca. $0.15 \mathrm{mmol}$ ), pentane-2,4-dione 5 (44 mg, $0.44 \mathrm{mmol}$ ) and sodium carbonate $(159 \mathrm{mg}, 1.50 \mathrm{mmol})$ in acetonitrile $\left(10 \mathrm{~cm}^{3}\right)$ was stirred for $48 \mathrm{~h}$ at $80{ }^{\circ} \mathrm{C}$. The mixture was cooled to RT and the solvent was evaporated under reduced pressure. The crude mixture was purified by dry flash chromatography using hexane-DCM (1:0 changing to 1:1) as eluent. The pure product was taken up in DCM-methanol and the solvents were evaporated under reduced pressure to leave the complex $13(158 \mathrm{mg}, \mathrm{ca} .74 \%)$ as a red powder. The ${ }^{1} \mathrm{H} \mathrm{NMR}\left(\mathrm{CDCl}_{3}\right)$ data are identical to that of the product from the reaction in refluxing 2-ethoxyethanol.

$\alpha, \omega$-Bis(4,4,5,5-tetramethyl-1,3,2-dioxaborolan-2-yl)poly(9,9-dioctyl-9H-fluorene-2,7-diyl) $16 \mathrm{a}$ and $\mathbf{1 6 b}$

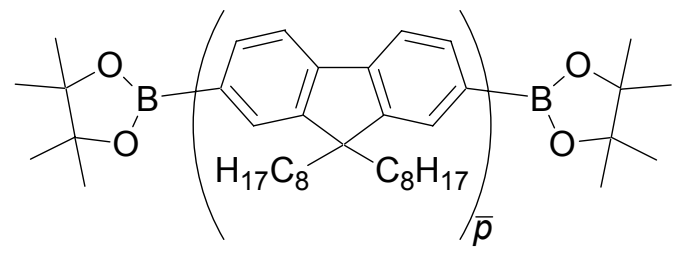

Aqueous tetraethylammonium hydroxide $\left(20 \% ; 10 \mathrm{~cm}^{3}, 13 \mathrm{mmol}\right)$ was added to a stirred solution of 9,9-dioctyl-2,7-bis(4,4,5,5-tetramethyl-1,3,2-dioxaborolan-2-yl)-9H-fluorene 14 (842 mg, $1.31 \mathrm{mmol}$ ), 2,7-dibromo-9,9-dioctyl-9H-fluorene 15, palladium acetate $(3 \mathrm{mg}, 13 \mu \mathrm{mol})$ and tricyclohexylphosphine $(18 \mathrm{mg}, 64 \mu \mathrm{mol})$ in toluene $\left(10 \mathrm{~cm}^{3}\right)$ at $\mathrm{RT}$. The mixture was stirred vigorously for $2 \mathrm{~h}$ at $90{ }^{\circ} \mathrm{C}$. The 
mixture was cooled to RT and added dropwise to methanol $\left(100 \mathrm{~cm}^{3}\right)$ stirred vigorously in an open vessel. The resultant precipitate was isolated, washed with methanol $\left(100 \mathrm{~cm}^{3}\right)$ and dried under vacuum.

Dibromide 15 (645 mg, $1.18 \mathrm{mmol})$. Crude macromonomer 16a (976 mg, ca. 98\%) as green fibers.

${ }^{1} \mathrm{H}$ NMR (500 MHz, $\left.\mathrm{CDCl}_{3}, \delta\right): 0.7-1.3\left(\right.$ br m, $\left.470 \mathrm{H}, 2 n \times\left(\mathrm{CH}_{2}\right)_{6} \mathrm{CH}_{3}\right), 1.41\left(\mathrm{~s}, 24 \mathrm{H}, 8 \times \mathrm{CH}_{3}\right) 2.13$ (br s, $\left.57 \mathrm{H}, 2 n \times \mathrm{CH}_{2}\right), 7.6-8.0($ br m, $93 \mathrm{H}, 6 n \times \mathrm{ArH})$.

Dibromide 15 (629 mg, $1.15 \mathrm{mmol}$ ). Crude macromonomer 16b (949 mg, ca. 95\%) as green fibers. ${ }^{1} \mathrm{H}$ NMR (400 MHz, $\left.\mathrm{CDCl}_{3}, \delta\right): 0.6-1.3\left(\right.$ br m, $\left.450 \mathrm{H}, 2 n \times\left(\mathrm{CH}_{2}\right)_{6} \mathrm{CH}_{3}\right), 1.41\left(\mathrm{~s}, 24 \mathrm{H}, 8 \times \mathrm{CH}_{3}\right) 2.12$ (br s, $\left.55 \mathrm{H}, 2 n \times \mathrm{CH}_{2}\right), 7.5-8.0($ br m, $86 \mathrm{H}, 6 n \times \mathrm{ArH})$.

The expected average degree of polymerization, $\bar{x}_{\mathrm{n}}$, was calculated using the equation: ${ }^{8}$

$$
\bar{x}_{\mathrm{n}}=(1+r) /(1+r-2 r p)
$$

where $p$ is a measure of the extent of the reaction (the proportion of functional groups that have participated in the polymerization reaction) and $r$ is the ratio of the monomers $\mathbf{1 4}$ and 15:

$$
r=[15] /[14]
$$

For a complete reaction, $p=1$, and the expected values of $\bar{x}_{\mathrm{n}}$ for the macromonomers 16a and $\mathbf{1 6} \mathbf{b}$ are:

$$
\begin{aligned}
& r(\mathbf{1 6 a})=1.18 \mathrm{mmol} / 1.31 \mathrm{mmol}=0.901(3 \text { s.f. }) \\
& \bar{x}_{\mathrm{n}}(\mathbf{1 6 a})=(1+0.901) /(1+0.901-(2 \times 0.901 \times 1))=19.2(3 \text { s.f. }) \\
& r(\mathbf{1 6 b})=1.15 \mathrm{mmol} / 1.31 \mathrm{mmol}=0.878(3 \text { s.f. }) \\
& \bar{x}_{\mathrm{n}}(\mathbf{1 6 b})=(1+0.878) /(1+0.878-(2 \times 0.878 \times 1))=15.4(3 \text { s.f. })
\end{aligned}
$$

The actual average degree of polymerization, $\bar{x}_{n}$, was estimated from proton NMR data. The methyl protons of the boronic ester end-groups are observed as a single peak at $\delta 1.41$. Assuming each chain is capped at both ends with a boronic ester group, the integral for this signal can be set to $24 \mathrm{H}$. The 
integral of the shielded alkyl protons of the octyl chains $\delta 0.6-1.3$ is divided by the number of shielded alkyl protons per 9,9-dioctyl-9H-fluorene-2,7-diyl repeat unit $(30 \mathrm{H})$ to provide as estimate for $\bar{x}_{\mathrm{n}}$ :

$$
\begin{aligned}
& \bar{x}_{\mathrm{n}}(\mathbf{1 6 a})=470 \mathrm{H} / 30 \mathrm{H}=16(2 \text { s.f. }) \\
& \bar{x}_{\mathrm{n}}(\mathbf{1 6 b})=450 \mathrm{H} / 30 \mathrm{H}=15(2 \text { s.f. })
\end{aligned}
$$

\section{$\alpha, \omega$-Bis(phenyl)poly[poly(9,9-dioctyl-9H-fluorene-2,7-diyl)-(9-\{N,N'-trans-bis[2-(2-pyridyl-}

\section{$\kappa \mathrm{N})$ benzo[b]thien-3-yl]iridium(III)(pentane-2,4-dionat-1-yl)\}-9-octyl-9H-fluorene-2,7-diyl)— poly(9,9-dioctyl-9H-fluorene-2,7-diyl)] 17a and 17b}

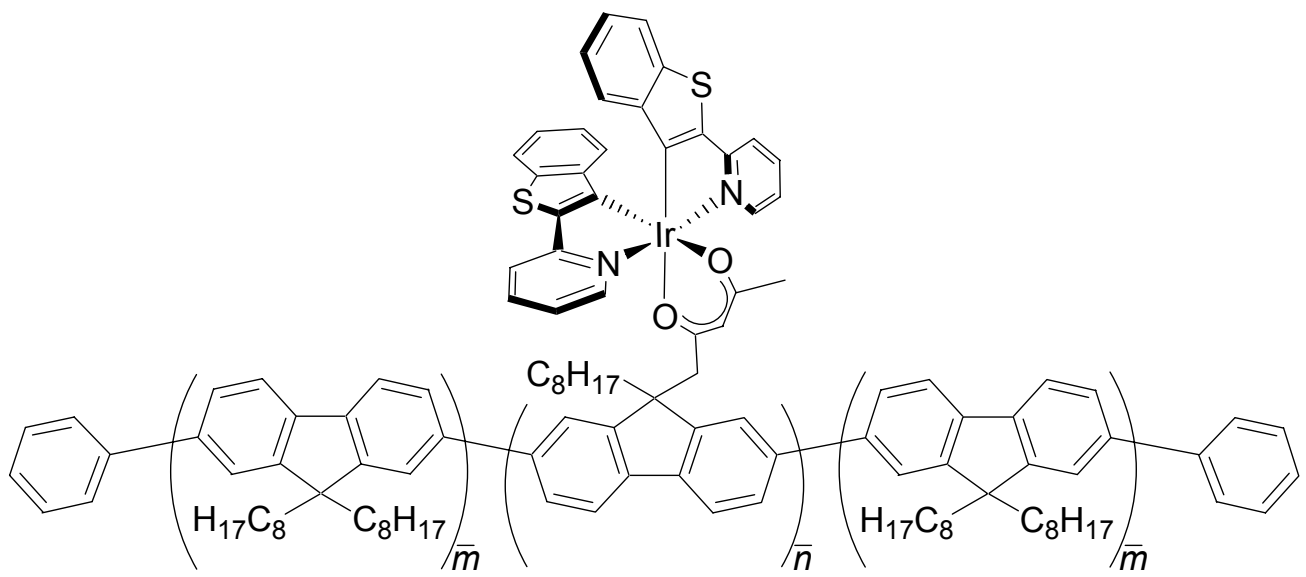

Aqueous tetraethylammonium hydroxide $\left(20 \% ; 4 \mathrm{~cm}^{3}, 5 \mathrm{mmol}\right)$ was added to a stirred mixture of [1(2,7-dibromo-9-octyl-9H-fluoren-9-yl)pentane-2,4-dionato]- $N, N^{\prime}$-trans-bis[2-(2-pyridyl- $\left.\kappa N\right)$ benzo[b]thien-3-yl]iridium(III) 12, crude $\alpha, \omega$-bis(4,4,5,5-tetramethyl-1,3,2-dioxaborolan-2-yl)poly(9,9-dioctyl$9 H$-fluorene-2,7-diyl) $\mathbf{1 6 a}$ or $\mathbf{1 6 b}$, palladium acetate $(1.2 \mathrm{mg}, 5.3 \mu \mathrm{mol})$ and tricyclohexylphosphine $(7.2$ $\mathrm{mg}, 26 \mu \mathrm{mol})$ in chlorobenzene $\left(4 \mathrm{~cm}^{3}\right)$ at RT. The mixture was stirred vigorously for $2 \mathrm{~h}$ at $90{ }^{\circ} \mathrm{C}$. The mixture was cooled to RT and added dropwise to methanol $\left(40 \mathrm{~cm}^{3}\right)$ stirred vigorously in an open vessel. The resultant precipitate was isolated, dissolved in chloroform and filtered and the filtrate was concentrated under reduced pressure. The chloroform solution was passed through a pad of silica twice, concentrated under reduced pressure and added dropwise to methanol $\left(40 \mathrm{~cm}^{3}\right)$ stirred vigorously in an 
open vessel. The resultant precipitate was isolated, washed with methanol $\left(40 \mathrm{~cm}^{3}\right)$ and dried under vacuum.

Crude macromonomer 16a (400 mg, ca. $54 \mu \mathrm{mol})$, spacerless monomer 12 (6.4 mg, $5.6 \mu \mathrm{mol})$. Spacerless copolymer 17a (307 mg, ca. 77\%) as yellow fibers.

Anal. Calcd for $\left[\left(\mathrm{C}_{6} \mathrm{H}_{5}\right)_{2}\left(\mathrm{C}_{29} \mathrm{H}_{40}\right)_{20.88}\left(\mathrm{C}_{52} \mathrm{H}_{45} \mathrm{IrN}_{2} \mathrm{O}_{2} \mathrm{~S}_{2}\right)_{0.12}\right]$ : C, 89.3; H, 10.2; N, 0.04. Found: C, 89.3; $\mathrm{H}, 10.2 ; \mathrm{N}, 0.09 . \mathrm{IR}\left(\mathrm{CHCl}_{3}\right) v_{\max }: 2929,2856,1458 \mathrm{~cm}^{-1} .{ }^{1} \mathrm{H}$ NMR $\left(400 \mathrm{MHz}, \mathrm{CDCl}_{3}, \delta\right): 0.6-1.4(\mathrm{br}$ m, $\left.580 \mathrm{H}, 2 n \times\left(\mathrm{CH}_{2}\right)_{6} \mathrm{CH}_{3}\right), 1.74(\mathrm{~s}, 0.2 \mathrm{H}$, pentyl 5-H $), 2.13\left(\right.$ br s, $\left.67 \mathrm{H}, 2 n \times \mathrm{CH}_{2}\right), 5.22(\mathrm{~s}, 0.06 \mathrm{H}$, pentyl 3-H), $6.04(\mathrm{~d}, J=9 \mathrm{~Hz}, 0.06 \mathrm{H}$, benzo 4-H or 7-H), $6.08(\mathrm{~d}, J=8 \mathrm{~Hz}, 0.06 \mathrm{H}$, benzo 4-H or 7-H), 6.42-7.20 (m, $2.7 \mathrm{H}, \mathrm{ArH}), 7.38(\mathrm{t}, J=7 \mathrm{~Hz}, 2.0 \mathrm{H}, 2 \times$ phenyl 4-H), $7.49(\mathrm{t}, J=7 \mathrm{~Hz}, 4.0 \mathrm{H}, 2 \times$ phenyl 3-H and 5-H), 7.5-8.0 (br m, $110 \mathrm{H}, 6 n \times \mathrm{ArH}) .{ }^{13} \mathrm{C} \mathrm{NMR}\left(\mathrm{CDCl}_{3}, \delta\right): 14.1(\mathrm{q}), 22.6(\mathrm{t}), 23.9(\mathrm{t})$, $29.2(\mathrm{t}), 30.0(\mathrm{t}), 31.8(\mathrm{t}), 40.4(\mathrm{t}), 55.3(\mathrm{~s}), 120.0(\mathrm{~d}), 121.5(\mathrm{~d}), 126.1(\mathrm{~d}), 127.2(\mathrm{~d}), 128.8(\mathrm{~d}), 140.0$ (s), 140.5 (s), 141.7 (s), 151.8 (s). MS-MALDI: $\bar{M}_{\mathrm{n}}$ 3200, $\bar{M}_{\mathrm{w}}$ 4100, PDI 1.3; $\mathrm{m} / z$ (\% number fraction, assignment): 1244 (4.58, $\left.P h-F_{3}-H\right), 1320$ (13.26, $\left.P h-F_{3}-P h\right), 1632\left(0.67, P h-F_{4}-H\right), 1945(0.37, H-$ $\left.F_{5}-H\right), 2021$ (5.96, Ph-F $\left.F_{5}-H\right), 2097$ (16.69, Ph-F $\left.-P h\right), 2115$ (0.39, unassigned), 2409 (0.79, Ph-F $F_{6}$ H), $2486\left(0.45, P h-F_{6}-P h\right), 2797$ (3.99, $\left.P h-F_{7}-H\right), 2873$ (12.61, $\left.P h-F_{7}-P h\right), 3186\left(0.62, P h-F_{8}-H\right)$, $3262\left(0.62, P h-F_{8}-P h\right), 3574\left(2.86, P h-F_{9}-H\right), 3650$ (9.39, $\left.P h-F_{9}-P h\right), 3962\left(0.50, P h-F_{10}-H\right), 4038$ (0.60, $\left.P h-F_{10}-P h\right), 4350\left(2.11, P h-F_{11}-H\right), 4426$ (6.35, Ph-F $\left.F_{11}-P h\right), 4737\left(0.34, P h-F_{12}-H\right), 4815$ (0.38, Ph-F $\left.F_{12}-P h\right), 5127\left(1.24, P h-F_{13}-H\right), 5203\left(4.49, P h-F_{13}-P h\right), 5513\left(0.32, P h-F_{14}-H\right), 5590$ (0.30, Ph-F $\left.F_{14}-P h\right), 5903\left(0.94, P h-F_{15}-H\right), 5979$ (3.21, Ph-F $\left.F_{15}-P h\right), 6366\left(0.27, P h-F_{16}-P h\right), 6678$ (0.54, Ph-F $\left.F_{17}-H\right), 6754\left(1.89, P h-F_{17}-P h\right), 7457\left(0.45, P h-F_{19}-H\right), 7531\left(1.37, P h-F_{19}-P h\right), 8309$ $\left(0.28, P h-F_{21}-P h\right), 9084\left(0.72, P h-F_{23}-P h\right), 9862\left(0.44, P h-F_{25}-P h\right)$. GPC (THF, $30{ }^{\circ} \mathrm{C}$, polystyrene reference): $\bar{M}_{\mathrm{n}} 17,000, M_{\mathrm{p}} 46,000, \bar{M}_{\mathrm{w}} 43,000$, PDI 2.6. DSC: $T_{\mathrm{g}} 60-68{ }^{\circ} \mathrm{C} ; T_{\mathrm{c}} 106{ }^{\circ} \mathrm{C} ; T_{\mathrm{m}} 129{ }^{\circ} \mathrm{C}$, $145{ }^{\circ} \mathrm{C}$. TGA: $T_{\mathrm{d}} 309{ }^{\circ} \mathrm{C}$ (the decomposition having an onset at $439{ }^{\circ} \mathrm{C}$, with the decomposition rate 
decreasing above $496{ }^{\circ} \mathrm{C}$ with $50 \%$ of the initial mass left and concluding at $796{ }^{\circ} \mathrm{C}$ to leave $0 \%$ of the initial mass).

Crude macromonomer 16b (400 mg, ca. $66 \mu \mathrm{mol})$, spacerless monomer 12 (19.7 mg, $17.1 \mu \mathrm{mol})$. Spacerless copolymer $\mathbf{1 7 b}$ (349 $\mathrm{mg}$, ca. 86\%) as orange fibers.

Anal. Calcd for $\left[\left(\mathrm{C}_{6} \mathrm{H}_{5}\right)_{2}\left(\mathrm{C}_{29} \mathrm{H}_{40}\right)_{20.64}\left(\mathrm{C}_{52} \mathrm{H}_{45} \mathrm{IrN}_{2} \mathrm{O}_{2} \mathrm{~S}_{2}\right)_{0.36}\right]$ : C, 88.6; H, 10.1; N, 0.12. Found: C, 88.6; $\mathrm{H}, 10.2 ; \mathrm{N}, 0.26 . \mathrm{IR}\left(\mathrm{CHCl}_{3}\right) v_{\max }: 2929,2856,1458 \mathrm{~cm}^{-1} .{ }^{1} \mathrm{H}$ NMR $\left(400 \mathrm{MHz}, \mathrm{CDCl}_{3}, \delta\right): 0.5-1.4(\mathrm{br}$ m, $\left.520 \mathrm{H}, 2 n \times\left(\mathrm{CH}_{2}\right)_{6} \mathrm{CH}_{3}\right), 1.74\left(\mathrm{~s}, 0.5 \mathrm{H}\right.$, pentyl 5- $\left.\mathrm{H}_{3}\right), 2.13\left(\right.$ br s, $\left.58 \mathrm{H}, 2 n \times \mathrm{CH}_{2}\right), 5.22(\mathrm{~s}, 0.2 \mathrm{H}$, pentyl 3-H), $6.03(\mathrm{~d}, J=8 \mathrm{~Hz}, 0.2 \mathrm{H}$, benzo 4-H or 7-H), 6.08 (d, $J=7 \mathrm{~Hz}, 0.2 \mathrm{H}$, benzo 4-H or 7-H), 6.43-7.22 (m, 3.8 H, ArH), 7.37 (t, $J=7 \mathrm{~Hz}, 2.1 \mathrm{H}, 2 \times$ phenyl 4-H), 7.49 (t, $J=7 \mathrm{~Hz}, 4.0 \mathrm{H}, 2 \times$ phenyl 3-H and 5-H), 7.5-8.1 (br m, $99 \mathrm{H}, 6 n \times \mathrm{ArH}), 8.30-8.35$ (m, 0.1 H, pyridyl 6-H). ${ }^{13} \mathrm{C}$ NMR $\left(\mathrm{CDCl}_{3}, \delta\right): 14.1(\mathrm{q}), 22.6(\mathrm{t}), 23.9(\mathrm{t}), 29.2(\mathrm{t}), 30.0(\mathrm{t}), 31.8(\mathrm{t}), 40.4(\mathrm{t}), 55.3(\mathrm{~s}), 120.0(\mathrm{~d}), 121.5(\mathrm{~d})$, 126.1 (d), 127.2 (d), 128.8 (d), 140.0 (s), 140.5 (s), 141.7 (s), 151.8 (s). MS-MALDI: $\bar{M}_{\mathrm{n}} 2800, \bar{M}_{\mathrm{w}}$ 3400, PDI 1.2; $m / z$ (\% number fraction, assignment): 1245 (5.40, $\left.P h-F_{3}-H\right), 1321\left(13.59, P h-F_{3}-P h\right)$, $1557\left(0.15, H-F_{4}-H\right), 1633$ (0.83, Ph-F $\left.-H\right), 1779$ (0.10, unassigned), 1803 (0.10, unassigned), 1945 (0.19, $\left.H-F_{5}-H\right), 2022$ (6.86, Ph-F $\left.-H\right), 2078$ (0.30, unassigned), 2097 (19.03, Ph-F $\left.-P h\right), 2118$ (0.14, unassigned), 2410 (0.63, Ph-F $\left.F_{6}-H\right), 2485$ (0.13, Ph-F $\left.-P h\right), 2722\left(0.33, H-F_{7}-H\right), 2798\left(5.47, P h-F_{7}-\right.$ H), 2842 (0.11, unassigned), 2874 (15.64, Ph-F $-P h), 3007$ (0.11, Ph-F $-I r-H), 3186\left(0.20, P h-F_{8}-\right.$ H), 3188 (0.12, unassigned), 3262 (0.45, Ph- $\left.F_{8}-P h\right), 3471$ (0.13, Ph-F $\left.-I r-P h\right), 3497\left(0.15, H-F_{9}-H\right)$, 3575 (3.44, Ph-F $-H$ ), 3629 (0.22, unassigned), 3651 (11.23, Ph-F $-P h), 3784$ (0.17, Ph-F $-I r-H)$, $3963\left(0.17, P h-F_{10}-H\right), 4038\left(0.43, P h-F_{10}-P h\right), 4043$ (0.10, unassigned), 4172 (0.19, Ph- $\left.F_{8}-I r-H\right)$, 4246 (0.14, $\left.P h-F_{8}-I r-P h\right), 4250$ (0.10, unassigned), 4351 (0.25, Ph-F $\left.F_{11}-H\right), 4427$ (6.48, $\left.P h-F_{11}-P h\right)$, $4739\left(0.20, P h-F_{12}-H\right), 4814\left(0.24, P h-F_{12}-P h\right), 4948\left(0.11, P h-F_{10}-I r-H\right), 5022\left(0.12, P h-F_{10}-I r-\right.$ $P h), 5127$ (1.10, Ph- $\left.F_{13}-H\right), 5202\left(0.25, P h-F_{13}-P h\right), 5800\left(0.48, P h-F_{12}-I r-P h\right), 5902\left(0.11, P h-F_{15}\right.$ H), 5907 (0.10, unassigned), 5980 (2.42, Ph-F $\left.F_{15}-P h\right), 6191\left(0.15, P h-F_{13}-I r-P h\right), 6756\left(1.25, P h-F_{17}-\right.$ 
Ph), 6770 (0.10, unassigned), $7533\left(0.27, P h-F_{19}-P h\right)$. GPC (THF, $30{ }^{\circ} \mathrm{C}$, polystyrene reference): $\bar{M}_{\mathrm{n}}$ 20,000, $M_{\mathrm{p}} 48,000, \bar{M}_{\mathrm{w}}$ 50,000, PDI 2.6. DSC: $T_{\mathrm{g}} 65-74{ }^{\circ} \mathrm{C} ; T_{\mathrm{c}} 115{ }^{\circ} \mathrm{C} ; T_{\mathrm{m}} 136{ }^{\circ} \mathrm{C}$. TGA: $T_{\mathrm{d}} 349{ }^{\circ} \mathrm{C}$ (the decomposition having an onset at $443{ }^{\circ} \mathrm{C}$, with the decomposition rate decreasing above $501{ }^{\circ} \mathrm{C}$ with $48 \%$ of the initial mass left to leave $43 \%$ of the initial mass at $1000{ }^{\circ} \mathrm{C}$ ).

The values for $\bar{x}_{\mathrm{n}}$, the structural variables $\bar{m}$ and $\bar{n}$, and the weight-percentage of the copolymer equivalent to the red-phosphorescent iridium complex 13 were estimated from proton NMR data. The meta protons of the phenyl end-groups are observed as a triplet at $\delta 7.49$. Assuming each chain is capped at both ends with a phenyl ring, the integral for this signal can be set to $4.0 \mathrm{H}(N . B$. The integral is uncorrected for the underlying fluorenyl aromatic protons. Although this omission has a significant effect on the values estimated for $\bar{x}_{\mathrm{n}}, \bar{m}$, and $\bar{n}$, the effect essentially cancels out when calculating the weight-percentage of the copolymer equivalent to the red-phosphorescent iridium complex 13). The integral of the shielded alkyl protons of the octyl chains $\delta 0.5-1.4$ is divided by the number of shielded alkyl protons per 9,9-dioctyl-9H-fluorene-2,7-diyl repeat unit $(30 \mathrm{H})$ to provide an estimate for $\bar{x}_{\mathrm{n}}$ :

$$
\begin{aligned}
& \bar{x}_{\mathrm{n}}(\mathbf{1 7 a})=576 \mathrm{H} / 30 \mathrm{H}=19(2 \text { s.f. }) \\
& \bar{x}_{\mathrm{n}}(\mathbf{1 7 b})=518 \mathrm{H} / 30 \mathrm{H}=17(2 \text { s.f. })
\end{aligned}
$$

The integral of the methine signal from the iridium-complex dionate ligand $(\delta 5.22)$ provides an estimate for $\bar{n}$ and thus $\bar{m}$ from the relation with $\bar{x}_{\mathrm{n}}$ :

$$
\begin{aligned}
& \bar{m}=\left(\bar{x}_{\mathrm{n}}-\bar{n}\right) / 2 \\
& \bar{n}(\mathbf{1 7 a})=0.06 \\
& \bar{m}(\mathbf{1 7 a})=[19(2 \text { s.f. })-0.06] / 2=9.6(2 \text { s.f. }) \\
& \bar{n}(\mathbf{1 7 b})=0.18 \\
& \bar{m}(\mathbf{1 7 b})=[17(2 \text { s.f. })-0.18] / 2=8.5(2 \text { s.f. })
\end{aligned}
$$


The weight-percentage of the copolymer equivalent to the red-phosphorescent iridium complex $\mathbf{1 3}$ can then be estimated from $\bar{n}$ and the molecular weights of 13 and the copolymers $17 \mathbf{a}$ and 17b (calculated using the values for $\bar{m}$ and $\bar{n}$ estimated from proton NMR data):

$$
\begin{aligned}
& \text { wt. } \% 13=\bar{n} \times \operatorname{MW}(\mathbf{1 3}) / \operatorname{MW}(\mathbf{1 7}) \\
& \text { wt. } \% 13(\mathbf{1 7 a})=0.06 \times 712 / 7647=0.6 \text { wt. } \%(1 \text { d.p. }) \\
& \text { wt. } \% 13(\mathbf{1 7 b})=0.18 \times 712 / 6977=1.8 \text { wt. } \%(1 \text { d.p. })
\end{aligned}
$$

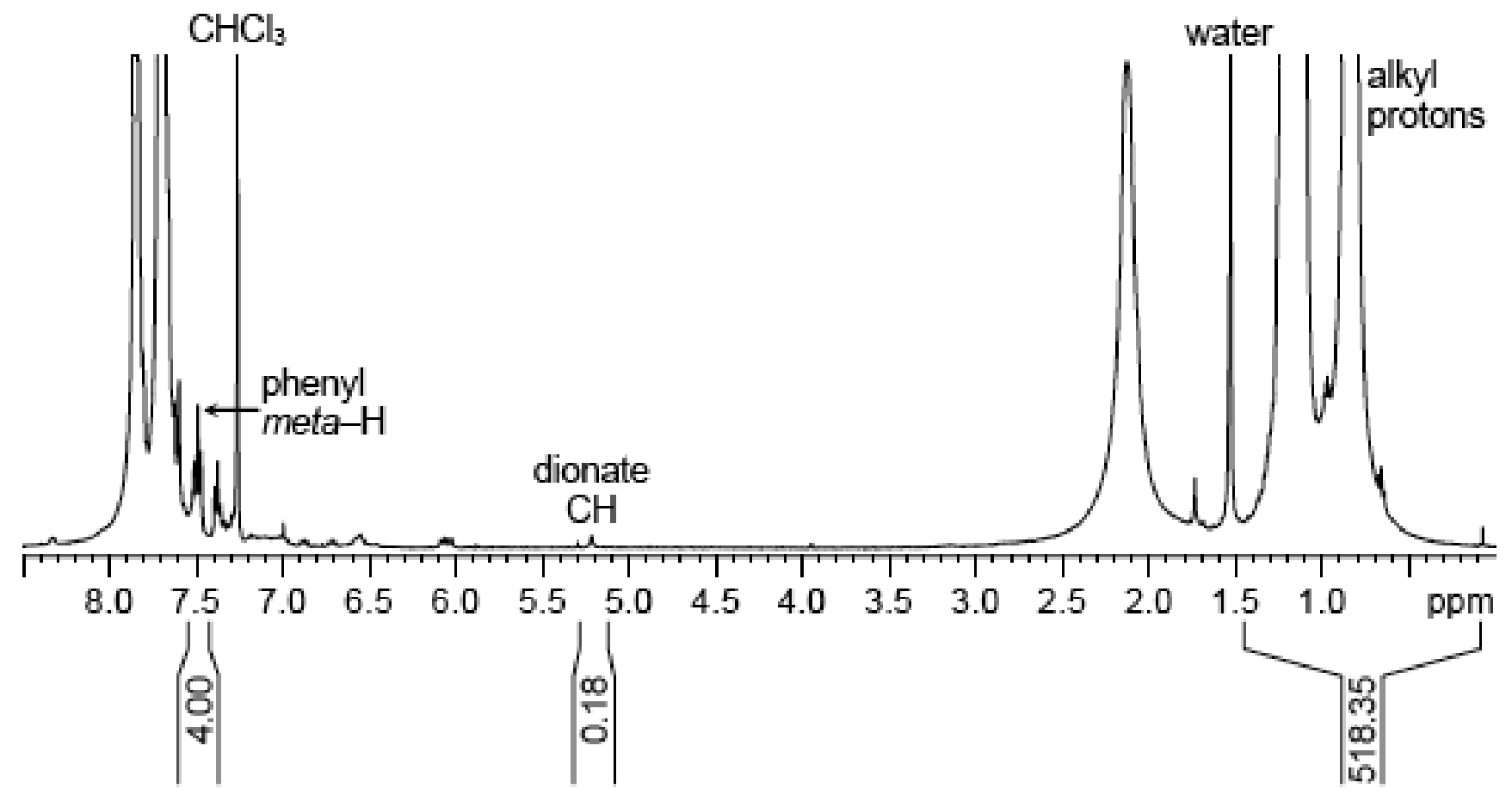

Figure S4. (a) Proton NMR spectrum (400 MHz, $d$-chloroform) of the spacerless copolymer 17b, scaled to show the end-group and iridium complex signals. Note the broadened dionate methine signal (cf. Figure S5). 


\section{$\alpha, \omega$-Bis(phenyl)poly[poly(9,9-dioctyl-9H-fluorene-2,7-diyl)—(9-\{N,N'-trans-bis[2-(2-pyridyl-} $\kappa N)$ benzo[b]thien-3-yl]iridium(III)(tridecane-2,4-dionat-13-yl)\}-9-octyl-9H-fluorene-2,7-diyl)poly(9,9-dioctyl-9H-fluorene-2,7-diyl)] 18a and 18b

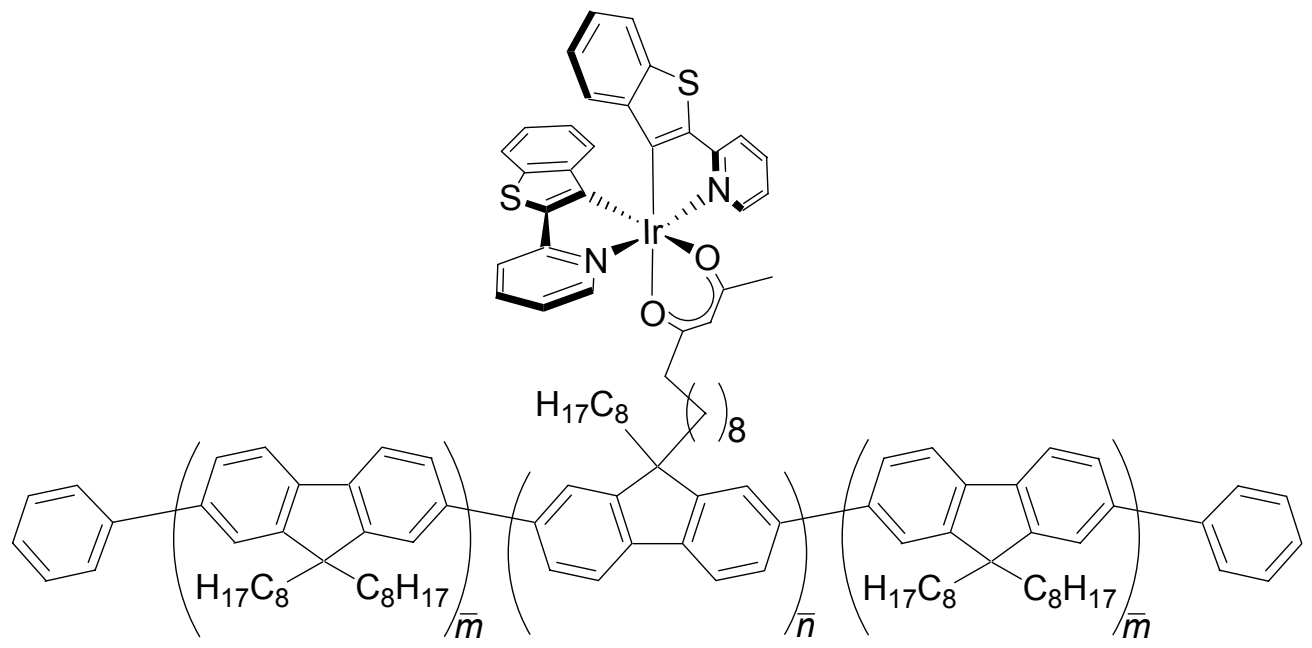

Aqueous tetraethylammonium hydroxide $\left(20 \% ; 4 \mathrm{~cm}^{3}, 5 \mathrm{mmol}\right)$ was added to a stirred mixture of [13(2,7-dibromo-9-octyl-9H-fluoren-9-yl)tridecane-2,4-dionato]- $N, N^{\prime}$-trans-bis[2-(2-pyridyl- $\left.\kappa N\right)$ benzo[b]thien-3-yl]iridium(III) 8 , crude $\alpha, \omega$-bis(4,4,5,5-tetramethyl-1,3,2-dioxaborolan-2-yl)poly(9,9-dioctyl9H-fluorene-2,7-diyl) $\mathbf{1 6 a}$ or $\mathbf{1 6 b}$, palladium acetate $(1.2 \mathrm{mg}, 5.3 \mu \mathrm{mol})$ and tricyclohexylphosphine (7.2 $\mathrm{mg}, 26 \mu \mathrm{mol})$ in chlorobenzene $\left(4 \mathrm{~cm}^{3}\right)$ at RT. The mixture was stirred vigorously for $2 \mathrm{~h}$ at $90{ }^{\circ} \mathrm{C}$. The mixture was cooled to RT and added dropwise to methanol $\left(40 \mathrm{~cm}^{3}\right)$ stirred vigorously in an open vessel. The resultant precipitate was isolated, dissolved in chloroform and filtered and the filtrate was concentrated under reduced pressure. The chloroform solution was passed through a pad of silica twice, concentrated under reduced pressure and added dropwise to methanol $\left(40 \mathrm{~cm}^{3}\right)$ stirred vigorously in an open vessel. The resultant precipitate was isolated, washed with methanol $\left(40 \mathrm{~cm}^{3}\right)$ and dried under vacuum. 
Crude macromonomer 16a (400 mg, ca. $54 \mu \mathrm{mol})$, tethered monomer 8 (7.1 mg, $5.6 \mu \mathrm{mol})$. Tethered copolymer 18a (303 mg, ca. 76\%) as yellow fibers.

Anal. Calcd for $\left[\left(\mathrm{C}_{6} \mathrm{H}_{5}\right)_{2}\left(\mathrm{C}_{29} \mathrm{H}_{40}\right)_{20.88}\left(\mathrm{C}_{60} \mathrm{H}_{61} \mathrm{IrN}_{2} \mathrm{O}_{2} \mathrm{~S}_{2}\right)_{0.12}\right]$ : C, 89.3; H, 10.2; N, 0.04. Found: C, 89.4; $\mathrm{H}, 10.2 ; \mathrm{N}, 0.11 . \mathrm{IR}\left(\mathrm{CHCl}_{3}\right) v_{\max }: 2929,2856,1458 \mathrm{~cm}^{-1} .{ }^{1} \mathrm{H}$ NMR $\left(400 \mathrm{MHz}, \mathrm{CDCl}_{3}, \delta\right): 0.6-1.4(\mathrm{br}$ m, $\left.580 \mathrm{H}, 2 n \times\left(\mathrm{CH}_{2}\right)_{6} \mathrm{CH}_{3}\right), 1.76\left(\mathrm{~s}, 0.2 \mathrm{H}\right.$, tridecyl 1- $\left.\mathrm{H}_{3}\right), 2.13\left(\right.$ br s, $\left.68 \mathrm{H}, 2 n \times \mathrm{CH}_{2}\right), 5.20(\mathrm{~s}, 0.08 \mathrm{H}$, tridecyl 3-H), $6.21(\mathrm{~d}, J=8 \mathrm{~Hz}, 0.1 \mathrm{H}$, benzo 4-H or 7-H), $6.28(\mathrm{~d}, J=9 \mathrm{~Hz}, 0.1 \mathrm{H}$, benzo 4-H or 7-H), 6.75-7.15 (m, $2.0 \mathrm{H}, \mathrm{ArH}), 7.38(\mathrm{t}, J=8 \mathrm{~Hz}, 2.4 \mathrm{H}, 2 \times$ phenyl 4-H), 7.49 (t, $J=8 \mathrm{~Hz}, 4.0 \mathrm{H}, 2 \times$ phenyl 3-H and 5-H), 7.5-8.0 (br m, $120 \mathrm{H}, 6 n \times \mathrm{ArH}), 8.38-8.42(\mathrm{~m}, 0.2 \mathrm{H}, 2 \times$ pyridyl $6-\mathrm{H}) .{ }^{13} \mathrm{C}$ $\operatorname{NMR}\left(\mathrm{CDCl}_{3}, \delta\right): 14.1(\mathrm{q}), 22.6(\mathrm{t}), 23.9(\mathrm{t}), 29.2(\mathrm{t}), 30.0(\mathrm{t}), 31.8(\mathrm{t}), 40.4(\mathrm{t}), 55.3(\mathrm{~s}), 119.9(\mathrm{~d}), 121.5$ (d), 126.1 (d), 127.2 (d), 128.8 (d), 140.0 (s), 140.5 (s), 141.7 (s), 151.8 (s). MS-MALDI: $\bar{M}_{\mathrm{n}} 3000$, $\bar{M}_{\mathrm{w}} 3700$, PDI 1.2; $\mathrm{m} / z$ (\% number fraction, assignment): $1244\left(4.77, P h-F_{3}-H\right), 1320\left(13.19, P h-F_{3}-\right.$ Ph), 1633 (0.96, Ph-F $-H), 1709$ (0.38, Ph-F $-P h), 1945$ (0.37, H-F $\left.F_{5}-H\right), 2021$ (5.56, Ph-F $\left.-H\right), 2077$ (0.39, unassigned), 2097 (18.36, Ph-F $-P h), 2409$ (0.64, Ph-F $-H), 2485$ (0.69, Ph-F $-P h), 2721$ (0.13, H-F $-H), 2797$ (4.33, Ph-F $-H), 2873$ (14.77, Ph-F $-P h), 2892$ (0.45, unassigned), 3185 (0.67, $\left.P h-F_{8}-H\right), 3261$ (0.66, $\left.P h-F_{8}-P h\right), 3573\left(2.42, P h-F_{9}-H\right), 3650$ (10.00, $\left.P h-F_{9}-P h\right), 3962(0.20, P h-$ $\left.F_{10}-H\right), 4037\left(0.62, P h-F_{10}-P h\right), 4350\left(2.29, P h-F_{11}-H\right), 4426\left(6.20, P h-F_{11}-P h\right), 4738\left(0.13, P h-F_{12}-\right.$ H), 4813 (0.44, $\left.P h-F_{12}-P h\right), 5126\left(1.48, P h-F_{13}-H\right), 5202\left(3.77, P h-F_{13}-P h\right), 5513\left(0.07, P h-F_{14}-H\right)$, $5590\left(0.22, P h-F_{14} P h\right), 5902\left(0.42, P h-F_{15}-H\right), 5978\left(2.45, P h-F_{15}-P h\right), 6292\left(0.09, P h-F_{16}-H\right)$, $6366\left(0.11, P h-F_{16}-P h\right), 6681\left(0.33, P h-F_{17}-H\right), 6754\left(1.35, P h-F_{17}-P h\right), 7530\left(0.76, P h-F_{19}-P h\right)$. GPC (THF, $30{ }^{\circ} \mathrm{C}$, polystyrene reference): $\bar{M}_{\mathrm{n}} 16,000, M_{\mathrm{p}} 38,000, \bar{M}_{\mathrm{w}} 42,000$, PDI 2.6. DSC: $T_{\mathrm{g}} 64-$ $73{ }^{\circ} \mathrm{C} ; T_{\mathrm{c}} 104{ }^{\circ} \mathrm{C} ; T_{\mathrm{m}} 128{ }^{\circ} \mathrm{C}, 145^{\circ} \mathrm{C}$. TGA: $T_{\mathrm{d}} 335{ }^{\circ} \mathrm{C}$ (the decomposition having an onset at $440{ }^{\circ} \mathrm{C}$, with the decomposition rate decreasing above $497{ }^{\circ} \mathrm{C}$ with $50 \%$ of the initial mass left and concluding at $795{ }^{\circ} \mathrm{C}$ to leave $0 \%$ of the initial mass). 
Crude macromonomer 16b (400 mg, ca. $66 \mu \mathrm{mol})$, tethered monomer $8(21.7 \mathrm{mg}, 17.2 \mu \mathrm{mol})$. Tethered copolymer $\mathbf{1 8 b}$ (318 mg, ca. 78\%) as orange fibers.

Anal. Calcd for $\left[\left(\mathrm{C}_{6} \mathrm{H}_{5}\right)_{2}\left(\mathrm{C}_{29} \mathrm{H}_{40}\right)_{20.64}\left(\mathrm{C}_{60} \mathrm{H}_{61} \mathrm{IrN}_{2} \mathrm{O}_{2} \mathrm{~S}_{2}\right)_{0.36}\right]$ : C, 88.6; H, 10.1; N, 0.12. Found: C, 88.8; $\mathrm{H}, 10.2 ; \mathrm{N}, 0.25 . \mathrm{IR}\left(\mathrm{CHCl}_{3}\right) v_{\max }: 2929,2856,1458 \mathrm{~cm}^{-1} .{ }^{1} \mathrm{H}$ NMR $\left(400 \mathrm{MHz}, \mathrm{CDCl}_{3}, \delta\right): 0.5-1.4(\mathrm{br}$ m, $\left.570 \mathrm{H}, 2 n \times\left(\mathrm{CH}_{2}\right)_{6} \mathrm{CH}_{3}\right), 1.76\left(\mathrm{~s}, 0.7 \mathrm{H}\right.$, tridecyl 1- $\left.\mathrm{H}_{3}\right), 2.13\left(\right.$ br s, $\left.69 \mathrm{H}, 2 n \times \mathrm{CH}_{2}\right), 5.20(\mathrm{~s}, 0.3 \mathrm{H}$, tridecyl 3-H), 6.21 (d, $J=8 \mathrm{~Hz}, 0.3 \mathrm{H}$, benzo 4-H or 7-H), 6.27 (d, $J=8 \mathrm{~Hz}, 0.3 \mathrm{H}$, benzo 4-H or 7-H), 6.75-7.09 (m, 2.9 H, ArH), 7.37 (t, $J=8 \mathrm{~Hz}, 2.4 \mathrm{H}, 2 \times$ phenyl 4-H), 7.49 (t, $J=8 \mathrm{~Hz}, 4.0 \mathrm{H}, 2 \times$ phenyl 3-H and 5-H), 7.5-8.1 (br m, $110 \mathrm{H}, 6 n \times \mathrm{ArH}), 8.37-8.42(\mathrm{~m}, 0.6 \mathrm{H}, 2 \times$ pyridyl $6-\mathrm{H}) .{ }^{13} \mathrm{C}$ $\operatorname{NMR}\left(\mathrm{CDCl}_{3}, \delta\right): 14.1(\mathrm{q}), 22.6(\mathrm{t}), 23.9(\mathrm{t}), 29.2(\mathrm{t}), 30.0(\mathrm{t}), 31.8(\mathrm{t}), 40.4(\mathrm{t}), 55.3(\mathrm{~s}), 119.9(\mathrm{~d}), 121.5$ (d), 126.1 (d), 127.2 (d), 128.8 (d), 140.0 (s), 140.5 (s), 141.7 (s), 151.8 (s). MS-MALDI: $\bar{M}_{\mathrm{n}} 2900$, $\bar{M}_{\mathrm{w}} 3600$, PDI $1.2 ; \mathrm{m} / z$ (\% number fraction, assignment): $1244\left(6.10, P h-F_{3}-H\right), 1320\left(12.97, P h-F_{3}-\right.$ Ph), 1632 (1.32, Ph-F $-H), 1708$ (0.58, Ph-F $-P h), 2021$ (7.07, Ph-F $-H), 2077$ (0.41, unassigned), 2097 (18.66, Ph-F $5-P h), 2409$ (0.41, $\left.P h-F_{6}-H\right), 2485$ (0.63, Ph-F 6 - Ph), 2797 (4.55, Ph-F $\left.-H\right), 2874$ (14.62, Ph-F $-P h), 3186\left(0.28, P h-F_{8}-H\right), 3262$ (0.57, $\left.P h-F_{8}-P h\right), 3574$ (2.97, Ph-F $\left.-H\right), 3650$ (9.84, $\left.P h-F_{9}-P h\right), 3963\left(0.34, P h-F_{10}-H\right), 4038\left(0.44, P h-F_{10}-P h\right), 4350$ (1.28, Ph-F $\left.{ }_{11}-H\right), 4426$ (5.73, Ph$\left.F_{11}-P h\right), 4443(0.28$, unassigned $), 4814\left(0.38, P h-F_{12}-P h\right), 5126\left(0.60, P h-F_{13}-H\right), 5202(3.79, P h-$ $\left.F_{13}-P h\right), 5590\left(0.20, P h-F_{14}-P h\right), 5910\left(0.34, P h-F_{12}-I r-P h\right), 5979$ (2.11, Ph-F $\left.15-P h\right), 6686(0.76$, $\left.P h-F_{14}-I r-P h\right), 6754\left(1.20, P h-F_{17}-P h\right), 7463\left(0.50, P h-F_{16}-I r-P h\right), 7530\left(0.62, P h-F_{19}-P h\right), 8307$ $\left(0.45, P h-F_{21}-P h\right) . \mathrm{GPC}\left(\mathrm{THF}, 30^{\circ} \mathrm{C}\right.$, polystyrene reference): $\bar{M}_{\mathrm{n}} 18,000, M_{\mathrm{p}} 48,000, \bar{M}_{\mathrm{w}} 48,000$, PDI 2.6. DSC: $T_{\mathrm{g}} 65-74{ }^{\circ} \mathrm{C} ; T_{\mathrm{c}} 109{ }^{\circ} \mathrm{C} ; T_{\mathrm{m}} 131{ }^{\circ} \mathrm{C}, 142{ }^{\circ} \mathrm{C}$. TGA: $T_{\mathrm{d}} 325{ }^{\circ} \mathrm{C}$ (the decomposition having an onset at $439{ }^{\circ} \mathrm{C}$, with the decomposition rate decreasing above $499{ }^{\circ} \mathrm{C}$ with $49 \%$ of the initial mass left to leave $41 \%$ of the initial mass at $1000{ }^{\circ} \mathrm{C}$ ). 
The values for $\bar{x}_{\mathrm{n}}$, the structural variables $\bar{m}$ and $\bar{n}$, and the weight-percentage of the copolymer equivalent to the red-phosphorescent iridium complex 13 were estimated from proton NMR data following the same method as described for the spacerless copolymers $\mathbf{1 7 .}$

$$
\begin{aligned}
& \bar{x}_{\mathrm{n}}(\mathbf{1 8 a})=581 \mathrm{H} / 30 \mathrm{H}=19(2 \text { s.f. }) \\
& \bar{n}(\mathbf{1 8 a})=0.08 \\
& \bar{m}(\mathbf{1 8 a})=[19(2 \text { s.f. })-0.08] / 2=9.6(2 \text { s.f. }) \\
& \text { wt. } \% \mathbf{1 3}(\mathbf{1 8 a})=0.08 \times 712 / 7742=0.7 \text { wt. } \%(1 \text { d.p. }) \\
& \bar{x}_{\mathrm{n}}(\mathbf{1 8 b})=568 \mathrm{H} / 30 \mathrm{H}=19(2 \text { s.f. }) \\
& \bar{n}(\mathbf{1 8 b})=0.30 \\
& \bar{m}(\mathbf{1 8 b})=[19(2 \text { s.f. })-0.30] / 2=9.3(2 \text { s.f. }) \\
& \text { wt. } \% \mathbf{1 3}(\mathbf{1 8 b})=0.30 \times 712 / 7730=2.8 \text { wt. } \%(1 \text { d.p. })
\end{aligned}
$$

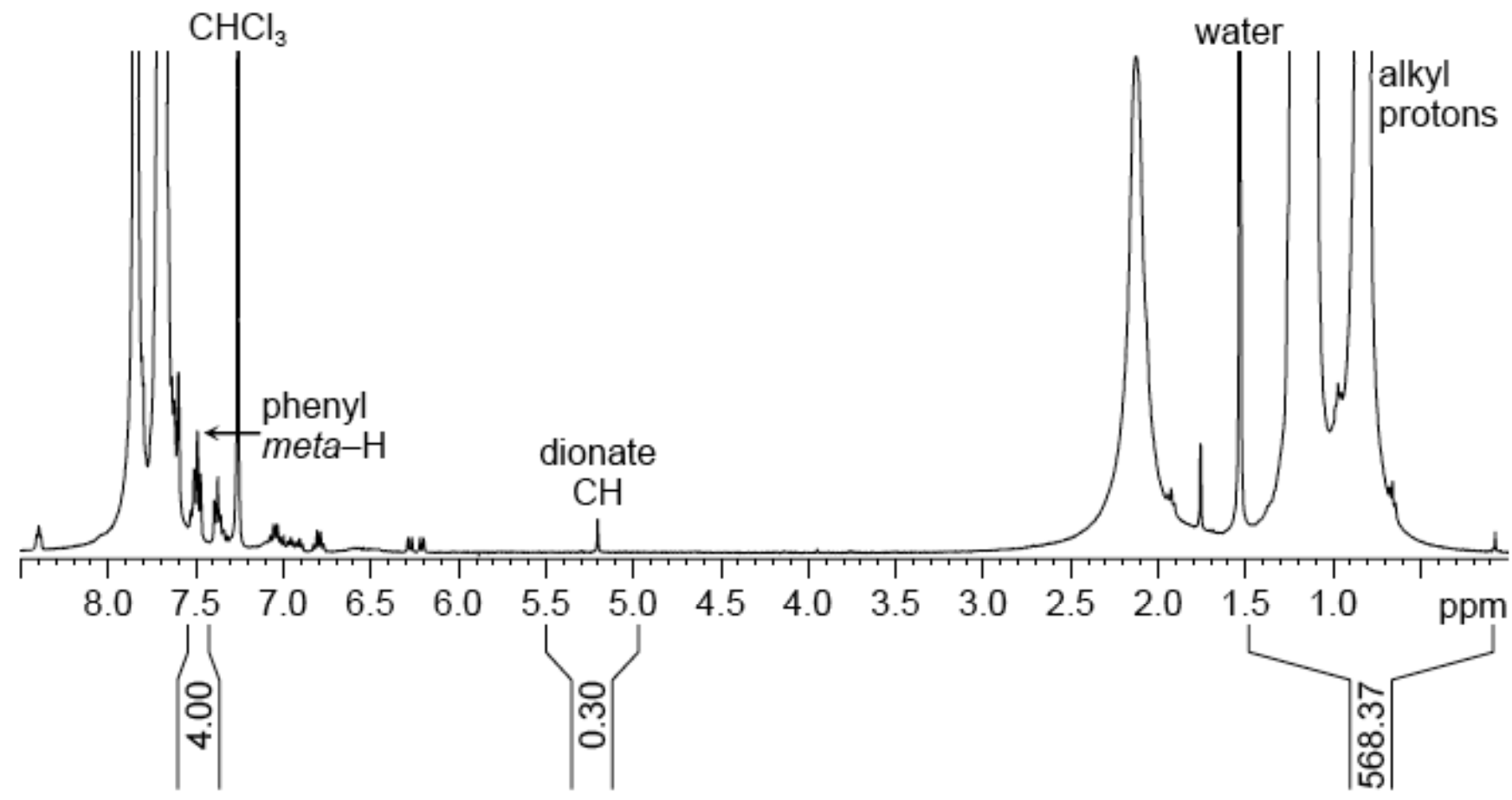

Figure S5. (a) Proton NMR spectrum (400 MHz, $d$-chloroform) of the tethered copolymer 18b, scaled to show the end-group and iridium complex signals. 
Triplet Energy Back Transfer in Conjugated Polymers with Pendant Phosphorescent Iridium Complexes - Supporting Information

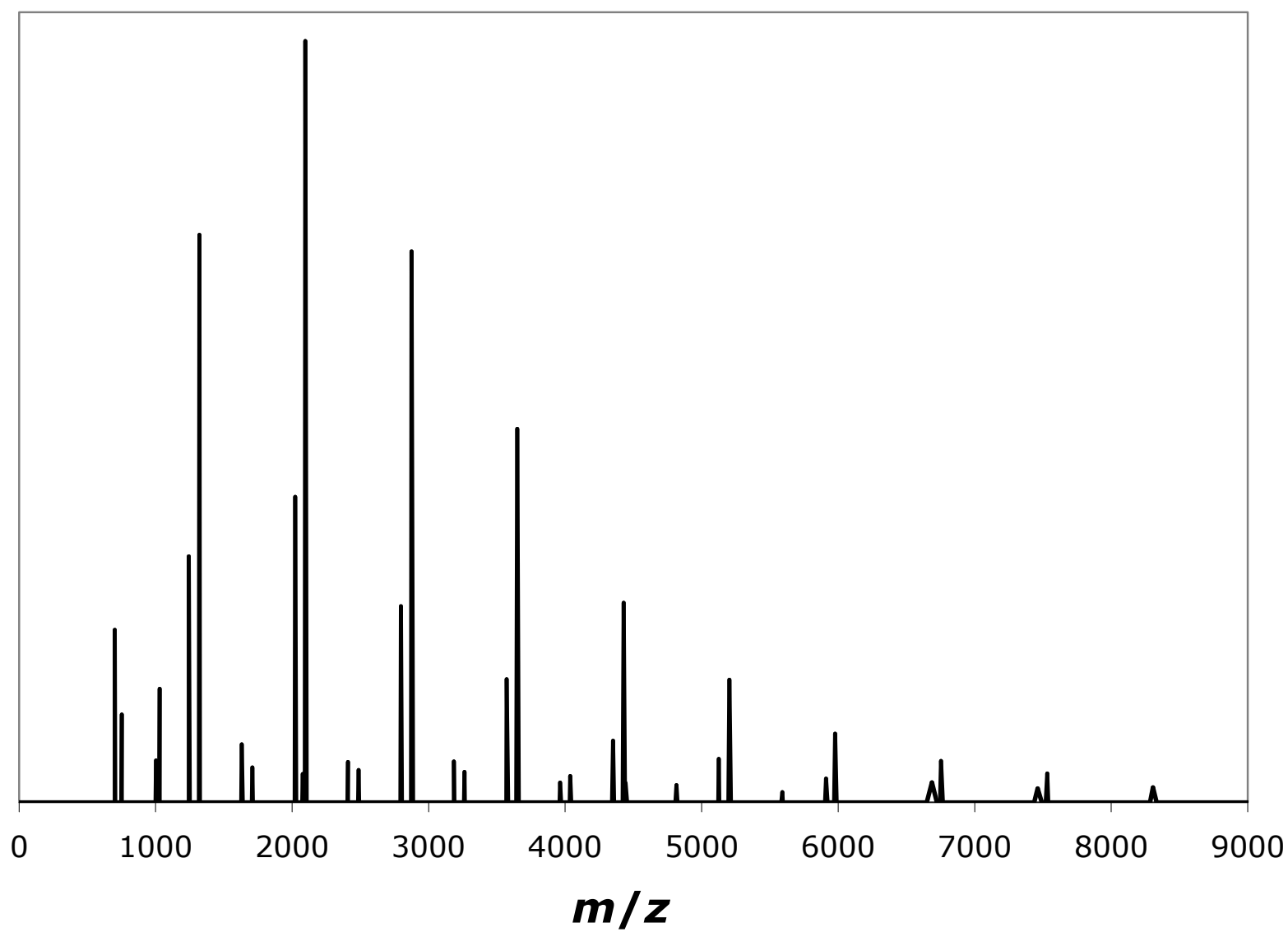

Figure S6. MALDI spectrum of the copolymer 18b, reproduced from peak data. 


\section{Optical Spectroscopy}

In contrast to the room temperature measurements, we observe no difference in the phosphorescence decay between the copolymers 17 and $\mathbf{1 8}$ at $20 \mathrm{~K}$.

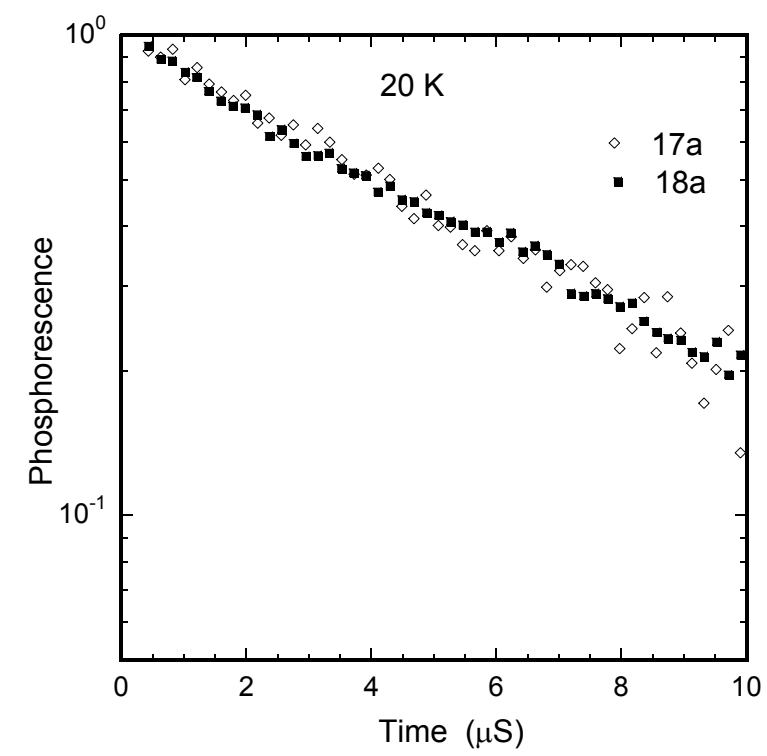

(a)

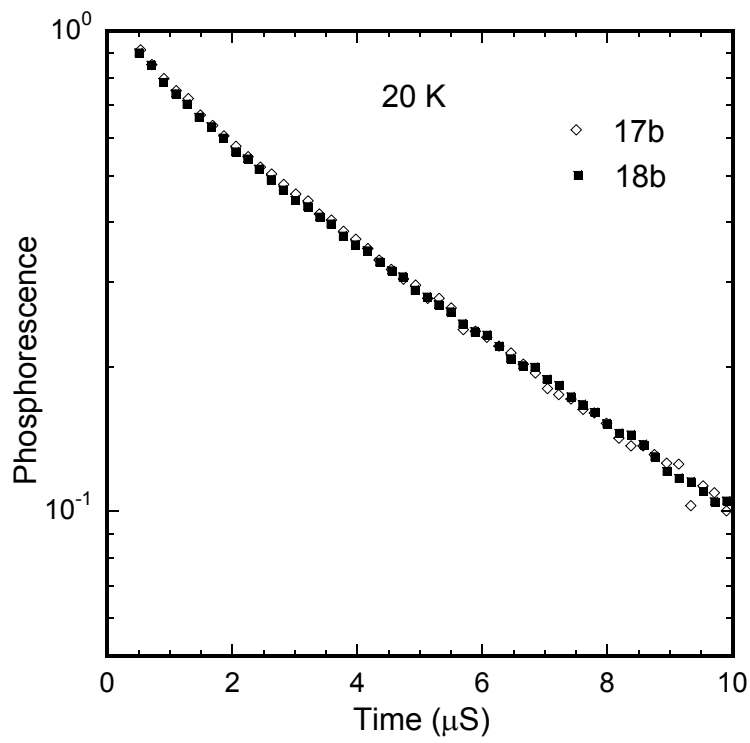

(b)

Figure S7. The decay of the photoluminescence intensity (excitation at $355 \mathrm{~nm}$ ) at $20 \mathrm{~K}$ from thin films of (a) the copolymers 17a and 18a (lower iridium-complex loading), and (b) the copolymers $\mathbf{1 7 b}$ and 18b (higher iridium-complex loading). 


\section{Luminescence quantum yields}

The overall photoluminescence quantum yields corresponding to Figure 4a are given in Table S1. It is evident that increasing the content of iridium complex in the copolymer increases the fluorescence quenching.

Table S1. Quantum yields for the overall photoluminescence and its contributions from fluorescence and phosporescence. The errors on the PL efficiencies are $\pm 4 \%$.

\begin{tabular}{lccccc}
\hline Copolymer & $\begin{array}{c}\text { Overall } \\
\text { PL } \\
\text { efficiency }\end{array}$ & $\begin{array}{c}\text { Fraction of } \\
\text { fluorescence }\end{array}$ & $\begin{array}{c}\text { Fluorescence } \\
\text { efficiency }\end{array}$ & $\begin{array}{c}\text { Fraction of } \\
\text { phosphorescence }\end{array}$ & $\begin{array}{c}\text { Phosphorescence } \\
\text { efficiency }\end{array}$ \\
\hline $\mathbf{1 7 a}$ & $19 \%$ & 0.37 & $6.9 \%$ & 0.63 & $11.7 \%$ \\
$\mathbf{1 8 a}$ & $32 \%$ & 0.30 & $9.6 \%$ & 0.70 & $22.4 \%$ \\
\hline $\mathbf{1 7 b}$ & $22 \%$ & 0.16 & $3.6 \%$ & 0.84 & $18.7 \%$ \\
$\mathbf{1 8 b}$ & $50 \%$ & 0.20 & $10.0 \%$ & 0.80 & $39.8 \%$ \\
\hline
\end{tabular}

Details of the electroluminescence measurements are given in Table S2. The errors on the EL quantum yields are $\pm 0.1 \%$. 
Table S2. The maximum EL Quantum yields along with the corresponding luminous efficiency, brightness, current densities, applied voltage and turn-on voltage.

\begin{tabular}{lcccccc}
\hline Copolymer & $\begin{array}{c}\text { Maximum } \\
\text { EL QY } \\
(\%)\end{array}$ & $\begin{array}{c}\text { Luminous } \\
\text { efficiency } \\
(\mathrm{cd} / \mathrm{A})\end{array}$ & $\begin{array}{c}\text { Brightness } \\
\left(\mathrm{cd} / \mathrm{m}^{2}\right)\end{array}$ & $\begin{array}{c}\text { Current } \\
\text { density } \\
\left(\mathrm{mA} / \mathrm{cm}^{2}\right)\end{array}$ & $\begin{array}{c}\text { Voltage for } \\
\text { max QE (V) }\end{array}$ & $\begin{array}{c}\text { Turn-on } \\
\text { Voltage } \\
(\mathrm{V})\end{array}$ \\
\hline $\mathbf{1 7 a}$ & 1.24 & 0.72 & 87.6 & 12.0 & 10.2 & 9.5 \\
$\mathbf{1 8 a}$ & 2.14 & 1.24 & 222 & 17.9 & 7.3 & 6.0 \\
\hline $\mathbf{1 7 b}$ & 1.7 & 1.0 & 165 & 16.8 & 13.0 & 12.0 \\
$\mathbf{1 8 b}$ & 2.0 & 1.15 & 87 & 7.6 & 10.0 & 9.5 \\
\hline
\end{tabular}

From Table S2 one can see that for copolymer 18b, the maximum EL quantum yield is obtained at much lower current densities than for the other three copolymers, which would be consistent with the occurrence of triplet-triplet annihilation in $\mathbf{1 8 b}$. For completeness, electroluminescence parameters are also given for the quantum yields at $100 \mathrm{~cd} / \mathrm{m}^{2}$ (Table S3) and for the quantum yields at a current density of $18 \mathrm{~mA} / \mathrm{cm}^{2}$ (Table S4).

Tables S3. The EL Quantum yields at $100 \mathrm{~cd} / \mathrm{m}^{2}$ along with the corresponding luminous efficiency, current densities, applied voltage.

\begin{tabular}{lcccc}
\hline Copolymer & $\begin{array}{c}\text { EL QY at } 100 \mathrm{~cd} / \mathrm{m}^{2} \\
(\%)\end{array}$ & $\begin{array}{c}\text { Luminous efficiency } \\
(\mathrm{cd} / \mathrm{A})\end{array}$ & $\begin{array}{c}\text { Current density } \\
\left(\mathrm{mA} / \mathrm{cm}^{2}\right)\end{array}$ & Voltage (V) \\
\hline $\mathbf{1 7 a}$ & 1.1 & 0.7 & 14.7 & 10.4 \\
$\mathbf{1 8 a}$ & 2.1 & 1.2 & 8.0 & 6.6 \\
\hline $\mathbf{1 7 b}$ & 1.7 & 1.0 & 10.5 & 12.4 \\
$\mathbf{1 8 b}$ & 2.0 & 1.1 & 9.1 & 10.2 \\
\hline
\end{tabular}


Tables S4. The brightness, external quantum efficiencies, luminance efficiencies and the corresponding voltages at a current density of $18 \mathrm{~mA} / \mathrm{cm}^{2}$.

\begin{tabular}{lcccc}
\hline Copolymer & $\begin{array}{c}\text { EL QY at } \\
18 \mathrm{~mA} / \mathrm{cm}^{2} \\
(\%)\end{array}$ & $\begin{array}{c}\text { Brightness } \\
\left(\mathrm{cd} / \mathrm{m}^{2}\right)\end{array}$ & $\begin{array}{c}\text { Luminance } \\
\text { Efficiency } \\
(\mathrm{cd} / \mathrm{A})\end{array}$ & $\begin{array}{c}\text { Voltage } \\
(\mathrm{V})\end{array}$ \\
\hline $\mathbf{1 7 a}$ & 1.2 & 126 & 0.71 & 10.6 \\
$\mathbf{1 8 a}$ & 2.1 & 222 & 1.20 & 7.3 \\
\hline $\mathbf{1 7 b}$ & 1.7 & 180 & 0.98 & 13.0 \\
$\mathbf{1 8 b}$ & 1.85 & 198 & 1.07 & 11.0 \\
\hline
\end{tabular}

\section{X-Ray Diffraction Studies}

Crystals of the spacerless monomer 12 were grown by slow evaporation of a DCM, diethyl ether and hexane solution of $\mathbf{1 2}$ (the crystals were observed unchanged up to $200{ }^{\circ} \mathrm{C}$ ). They were small and weakly diffracting, and a synchrotron radiation source was used to collect diffraction data. Data were collected at Station 9.8, Daresbury SRS, UK, using a Bruker SMART CCD diffractometer. A single crystal of 12 was selected under inert atmosphere, encased in perfluoro-polyether oil, and mounted on the end of a glass fiber. The fiber, secured on a goniometer head was then placed under a stream of cold nitrogen maintained at $150 \mathrm{~K}$.

Crystals of the complex 13 were grown by slow diffusion of diethyl ether vapor into a DCM solution of 13 (the crystals blackened at $339{ }^{\circ} \mathrm{C}$ and no further change was observed up to $400{ }^{\circ} \mathrm{C}$ ). A single crystal was mounted on a glass fiber using perfluoropolyether oil and cooled rapidly to $150 \mathrm{~K}$ in a stream of cold nitrogen using an Oxford Cryosystems CRYOSTREAM unit. Diffraction data were measured using an Enraf-Nonius KappaCCD diffractometer. Intensity data were processed using the DENZO-SMN package. ${ }^{9}$

The structures of $\mathbf{1 2}$ and $\mathbf{1 3}$ were solved using the direct-methods program SIR92, ${ }^{10}$ which located all non-hydrogen atoms and refined by full-matrix least squares procedure on F. Refinement and graphical 
calculations were performed using the CRYSTALS program suite. ${ }^{11}$ All hydrogen atoms were generated and allowed to ride on their corresponding carbon atoms with fixed thermal parameters. Hydrogen atoms were positioned geometrically after each cycle of refinement. A 3-term Chebychev polynomial weighting scheme was applied in both cases. The asymmetric unit contains 1 molecule of $\mathrm{CH}_{3} \mathrm{CN}$ disordered over a center of symmetry that was refined with restrains. Figure S7 contains the ORTEP diagram (40\% probability) for complex 13, Table S5 lists significant molecular parameters for the structures of 12 and $\mathbf{1 3}$ and Table S6 contains crystallographic data for compounds $\mathbf{1 2}$ and $\mathbf{1 3 .}$

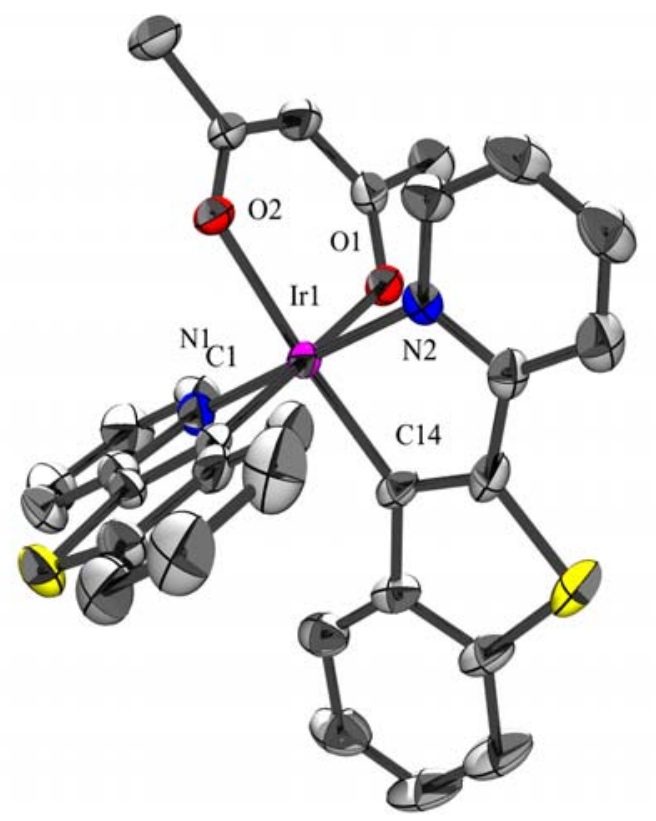

Figure S8. X-ray crystal structure of the complex 13, showing the pertinent atom labels. Displacement ellipsoids are drawn at the $40 \%$ probability level. Hydrogen atoms and solvent have been omitted for clarity. 
Table S5. Significant Molecular Parameters for the Structures of 12 and 13

\begin{tabular}{|c|c|c|c|c|}
\hline \multirow{2}{*}{$\begin{array}{l}\text { Compound } \\
\mathbf{1 2}\end{array}$} & \multicolumn{2}{|c|}{ Bond Lengths $(\AA)$} & \multicolumn{2}{|l|}{ Bond Angles $\left({ }^{\circ}\right)$} \\
\hline & $\operatorname{Ir}(1)-\mathrm{N}(1)$ & $2.046(3)$ & $\mathrm{N}(1)-\operatorname{Ir}(1)-\mathrm{N}(2)$ & 178.81(9) \\
\hline & $\operatorname{Ir}(1)-\mathrm{N}(2)$ & $2.057(2)$ & $\mathrm{N}(1)-\operatorname{Ir}(1)-\mathrm{O}(1)$ & $93.53(13)$ \\
\hline & $\operatorname{Ir}(1)-\mathrm{O}(1)$ & $2.121(3)$ & $\mathrm{N}(2)-\operatorname{Ir}(1)-\mathrm{O}(1)$ & $85.61(13)$ \\
\hline & $\operatorname{Ir}(1)-\mathrm{O}(2)$ & $2.117(3)$ & $\mathrm{N}(1)-\mathrm{Ir}(1)-\mathrm{O}(2)$ & $87.21(13)$ \\
\hline & $\operatorname{Ir}(1)-C(1)$ & $1.994(4)$ & $\mathrm{N}(2)-\mathrm{Ir}(1)-\mathrm{O}(2)$ & $93.61(13)$ \\
\hline & $\operatorname{Ir}(1)-C(14)$ & $1.992(4)$ & $\mathrm{O}(1)-\operatorname{Ir}(1)-\mathrm{O}(2)$ & $89.79(12)$ \\
\hline & & & $\mathrm{N}(1)-\operatorname{Ir}(1)-\mathrm{C}(1)$ & $80.74(16)$ \\
\hline & & & $\mathrm{N}(2)-\operatorname{Ir}(1)-\mathrm{C}(1)$ & $100.11(16)$ \\
\hline & & & $\mathrm{O}(1)-\operatorname{Ir}(1)-\mathrm{C}(1)$ & $174.22(15)$ \\
\hline & & & $\mathrm{O}(2)-\operatorname{Ir}(1)-\mathrm{C}(1)$ & $90.69(12)$ \\
\hline & & & $\mathrm{N}(1)-\operatorname{Ir}(1)-\mathrm{C}(14)$ & $99.22(16)$ \\
\hline & & & $\mathrm{N}(2)-\operatorname{Ir}(1)-\mathrm{C}(14)$ & $79.99(17)$ \\
\hline & & & $\mathrm{O}(1)-\operatorname{Ir}(1)-\mathrm{C}(14)$ & $91.27(12)$ \\
\hline & & & $\mathrm{C}(1)-\operatorname{Ir}(1)-\mathrm{C}(14)$ & $88.91(18)$ \\
\hline & & & $\mathrm{O}(2)-\operatorname{Ir}(1)-\mathrm{C}(14)$ & $173.41(16)$ \\
\hline \multirow[t]{15}{*}{13} & $\operatorname{Ir}(1)-\mathrm{O}(1)$ & $2.103(4)$ & $\mathrm{O}(1)-\operatorname{Ir}(1)-\mathrm{O}(2)$ & $88.71(16)$ \\
\hline & $\operatorname{Ir}(1)-\mathrm{O}(2)$ & $2.124(4)$ & $\mathrm{O}(1)-\operatorname{Ir}(1)-\mathrm{N}(1)$ & $85.7(2)$ \\
\hline & $\operatorname{Ir}(1)-\mathrm{N}(1)$ & $2.048(4)$ & $\mathrm{O}(2)-\operatorname{Ir}(1)-\mathrm{N}(1)$ & 93.21(19) \\
\hline & $\operatorname{Ir}(1)-\mathrm{N}(2)$ & $2.051(4)$ & $\mathrm{O}(1)-\operatorname{Ir}(1)-\mathrm{N}(2)$ & 93.64(19) \\
\hline & $\operatorname{Ir}(1)-C(1)$ & $2.015(6)$ & $\mathrm{O}(2)-\operatorname{Ir}(1)-\mathrm{N}(2)$ & $85.94(19)$ \\
\hline & $\operatorname{Ir}(1)-C(14)$ & $1.982(7)$ & $\mathrm{N}(1)-\operatorname{Ir}(1)-\mathrm{N}(2)$ & $179.0(3)$ \\
\hline & & & $\mathrm{O}(1)-\operatorname{Ir}(1)-\mathrm{C}(1)$ & $89.15(18)$ \\
\hline & & & $\mathrm{O}(2)-\operatorname{Ir}(1)-\mathrm{C}(1)$ & $174.1(2)$ \\
\hline & & & $N(1)-\operatorname{Ir}(1)-C(1)$ & $81.1(2)$ \\
\hline & & & $\mathrm{N}(2)-\operatorname{Ir}(1)-\mathrm{C}(1)$ & $99.7(2)$ \\
\hline & & & $\mathrm{O}(1)-\operatorname{Ir}(1)-\mathrm{C}(14)$ & $173.9(3)$ \\
\hline & & & $\mathrm{O}(2)-\operatorname{Ir}(1)-\mathrm{C}(14)$ & $93.21(17)$ \\
\hline & & & $\mathrm{N}(1)-\operatorname{Ir}(1)-\mathrm{C}(14)$ & $100.0(3)$ \\
\hline & & & $\mathrm{N}(2)-\operatorname{Ir}(1)-\mathrm{C}(14)$ & $80.7(3)$ \\
\hline & & & $\mathrm{C}(1)-\operatorname{Ir}(1)-\mathrm{C}(14)$ & $89.5(3)$ \\
\hline
\end{tabular}


Table S6. Crystallographic Data for Compounds 12 and 13

\begin{tabular}{|c|c|c|}
\hline Compound & 12 & 13 \\
\hline Formula & $\mathrm{C}_{52} \mathrm{H}_{45} \mathrm{Br}_{2} \mathrm{IrN}_{2} \mathrm{O}_{2} \mathrm{~S}_{2}+\mathrm{C}_{2} \mathrm{H}_{3} \mathrm{~N}$ & $\mathrm{C}_{31} \mathrm{H}_{23} \mathrm{IrN}_{2} \mathrm{O}_{2} \mathrm{~S}_{2}+\mathrm{C}_{4} \mathrm{H}_{10} \mathrm{O}$ \\
\hline Crystal Class & Orthorhombic & Orthorhombic \\
\hline \multicolumn{3}{|l|}{ Cell parameters } \\
\hline $\mathrm{a}$ & $46.929(4)$ & $12.6135(2)$ \\
\hline $\mathrm{b}$ & $11.5729(9)$ & $14.2569(2)$ \\
\hline c & $17.8941(14)$ & $18.0180(3)$ \\
\hline Space Group & I b a 2 & P c a 21 \\
\hline Volume & $9718.4(14)$ & $3240.17(9)$ \\
\hline Z & 8 & 4 \\
\hline Radiation type & synchrotron & Mo K\a \\
\hline Wavelength & 0.6904 & 0.710730 \\
\hline $\mathrm{Dx}$ & 1.623 & 1.61 \\
\hline $\mathrm{Mr}$ & 1187.15 & 786.01 \\
\hline$\mu$ & 4.522 & 4.287 \\
\hline Temperature (K) & 150 & 180 \\
\hline Size & $0.02 \times 0.04 \times 0.04$ & $0.05 \times 0.12 \times 0.32$ \\
\hline Colour / Shape & yellow / needle & orange / plate \\
\hline Reflections measured & 15096 & 27296 \\
\hline Theta range & 2.20 to 24 & 5 to 27 \\
\hline Absorption type & multi-scan & multi-scan \\
\hline Independent reflections (Rint) & $17842(0.02)$ & $6707(0.03)$ \\
\hline Hmin, Hmax & $-55,55$ & $-15,16$ \\
\hline $\mathrm{Kmin}, \mathrm{Kmax}$ & $-13,13$ & $-18,18$ \\
\hline Lmin, Lmax & $-21,21$ & $-18,23$ \\
\hline R-factor & 0.0388 & 0.020 \\
\hline Weighted R-factor & 0.0455 & 0.028 \\
\hline Reflections used / I > 3.00 $\sigma(\mathrm{I})$ & 4363 & 5758 \\
\hline Delta Rho min / Delta Rho max & $-3.68 / 8.27$ & $-1.48 / 1.06$ \\
\hline Number of parameters & 551 & 389 \\
\hline Goodness of fit & 1.033 & 0.846 \\
\hline
\end{tabular}




\section{References}

(1) Armarego, W. L. F.; Perrin, D. D. Purification of Laboratory Chemicals, Butterworth-Heinemann, Oxford, United Kingdom, 1996, fourth edition.

(2) Lamansky, S.; Djurovich, P.; Murphy, D.; Abdel-Razzaq, F.; Lee, H.-E.; Adachi, C.; Burrows, P. E.; Forrest, S. R.; Thompson, M. E. J. Am. Chem. Soc. 2001, 123, 4304-4312.

(3) Ranger, M.; Leclerc, M. Can. J. Chem. 1998, 76, 1571-1577.

(4) Effenberger, F.; Schönwälder, K.-H. Chem. Ber. 1984, 117, 3270-3279.

(5) Harwood, L. M.; Moody, C. J. Experimental Organic Chemistry: Principles and Practice, Blackwell Scientific Publications, Oxford, United Kingdom, 1989, first edition.

(6) Ego, C.; Marsitzky, D.; Becker, S.; Zhang, J.; Grimsdale, A. C.; Müllen, K.; MacKenzie, J. D.; Silva, C.; Friend, R. H. J. Am. Chem. Soc. 2003, 125, 437-443.

(7) Bloomer, J. L.; Zaidi, S. M. H.; Strupczewski, J. T.; Brosz, C. S.; Gudzyk, L. A. J. Org. Chem. 1974, 39, 3615-3616.

(8) Flory, P. J. Principles of Polymer Chemistry, Cornell University Press, Ithaca, 1953.

(9) Otwinowski, Z.; Minor, W. Methods Enzymol., 1997, 276, 307-326, Processing of X-ray Diffraction Data Collected in Oscillation Mode, Eds. Carter, C. W.; Sweet, R. M., Academic Press.

(10) Altomare, A.; Cascarano, G.; Giacovazzo, C.; Guagliardi, A.; Burla, M. C.; Polidori, G.; Camalli, M. J. Appl. Crystallogr. 1994, 27, 435.

(11) Watkin, D. J.; Prout, C. K.; Carruthers, J. R.; Betteridge, P. W.; Cooper, R. I. CRYSTALS issue 11, 2001, Chemical Crystallography Laboratory, Oxford, UK, 